\title{
Classification of low-luminosity stellar X-ray sources in the field of the Draco dwarf spheroidal galaxy ${ }^{\star}$
}

\author{
Sara Saeedi ${ }^{1}$, Manami Sasaki ${ }^{2}$, Beate Stelzer ${ }^{1,3}$, and Lorenzo Ducci ${ }^{1}$ \\ ${ }^{1}$ Institut für Astronomie und Astrophysik, Kepler Center for Astro and Particle Physics, Eberhard Karls Universität Tübingen, \\ Sand 1, 72076 Tübingen, Germany \\ e-mail: saeedi@astro.uni-tuebingen.de \\ 2 Dr. Karl Remeis Observatory and ECAP, Universität Erlangen-Nürnberg, Sternwartstr. 7, 96049 Bamberg, Germany \\ 3 INAF - Osservatorio Astronomico di Palermo, Piazza del Parlamento 1, 90134 Palermo, Italy
}

Received 26 December 2018 / Accepted 20 May 2019

\begin{abstract}
Aims. A previous study of the X-ray luminosity function of the X-ray sources in the Draco dwarf spheroidal (dSph) galaxy field indicates the presence of a population of unknown X-ray sources in the soft energy range of $0.5-2 \mathrm{keV}$. In 2015 , there were twenty-six further deep XMM-Newton observations of Draco dSph, providing an opportunity for a new study of the as yet unclassified sources. Methods. We applied the classification criteria presented in our previous multi-wavelength study of the X-ray sources of the Draco dSph to the sources detected in the combined 2009 and 2015 XMM-Newton data set. These criteria are based on X-ray studies and properties of the optical, near-infrared, and mid-infrared counterparts and allows us to distinguish background active galactic nuclei (AGNs) and galaxies from other types of X-ray sources. In this work we performed X-ray spectral and timing analyses for fifteen sources in the field of Draco dSph with stellar counterparts.

Results. We present the classification of X-ray sources, for which the counterpart is identified as a stellar object based on our criteria from multi-wavelength data. We identify three new symbiotic stars in the Draco dSph with X-ray luminosities between $\sim 3.5 \times 10^{34} \mathrm{erg} \mathrm{s}^{-1}$ and $5.5 \times 10^{34} \mathrm{erg} \mathrm{s}^{-1}$. The X-ray spectral analysis shows that two of the classified symbiotic stars are $\beta$-type. This is the first identification of this class of symbiotic stars in a nearby galaxy. Eight sources are classified as Galactic M dwarfs in the field of the Draco dSph. These Mdwarfs are between $\sim 140$ and $800 \mathrm{pc}$ distant, with X-ray luminosities are between $10^{28}$ and $10^{29} \mathrm{erg} \mathrm{s}^{-1}$ and logarithmic ratios of X-ray to bolometric luminosity, $\log \left(\frac{L_{\mathrm{X}}}{L_{\mathrm{bol}}}\right)$, between -3.4 and -2.1 . The multiple observations allowed us to investigate flare activity of the Mdwarfs. For $5 \mathrm{Mdwarfs}$ flare(s) are observed with a significance of $>3 \sigma$ level of confidence. Moreover, we classified three foreground sources, located at distances of the order of $\sim 1-3 \mathrm{kpc}$ in the field of the Draco dSph. Based on both the X-ray luminosities of these foreground sources $\left(>10^{30} \mathrm{erg} \mathrm{s}^{-1}\right)$ and their optical counterparts (late type $\mathrm{G}$ or $\mathrm{K}$ stars), these $\mathrm{X}$-ray sources are classified as candidates of contact binary systems.

Conclusions. Our study of X-ray sources of the Draco dSph shows that accreting white dwarfs are the most promising X-ray population of dSphs, which is in line with theoretical expectations. The number of Galactic M dwarfs detected at our X-ray sensitivity limit is consistent with the expectation based on the space density of M dwarfs.
\end{abstract}

Key words. galaxies: dwarf - X-rays: galaxies - X-rays: binaries - X-rays: stars - binaries: symbiotic - stars: mass-loss

\section{Introduction}

Most of the satellite galaxies of our Milky Way are dwarf spheroidal galaxies ( $\mathrm{dSph}$ ), which are faint with luminosities of $10^{5-7} L_{\odot}$ and have an approximately spherical shape. Observations of nearby dwarf galaxies show that most are metal-poor systems with metallicities as low as $[\mathrm{Fe} / \mathrm{H}]<-3$ (e.g. Kirby et al. 2008; Aoki et al. 2009; Frebel et al. 2010). As many dSphs show no recent star formation, they are ideal targets to study the old stellar populations in galaxies formed in the early stages of galaxy evolution. However, in comparison to other nearby galaxies, the X-ray population of low-mass satellite galaxies of the Milky Way (e.g. dSphs) is poorly studied (e.g. Fabbiano 2006).

Theoretically, the old stellar population of the dSphs makes the presence of X-ray binaries (XRBs) very unlikely. Because these compact objects form at the end of the life of massive stars, XRBs with high-mass companions (high-mass X-ray bina-

\footnotetext{
* Based on observations obtained with XMM-Newton, an ESA science mission with instruments and contributions directly funded by ESA Member States and NASA.
}

ries, $\mathrm{HMXBs}$ ) are often found in very young stellar populations. Additionally, the presence of X-ray binaries with low-mass companion stars (low-mass X-ray binaries, LMXBs) in a dSph is a theoretical challenge. LMXBs are supposed to form a few Gyr after the general star formation bursts in galaxies. Since the compact object consumes the mass of the less-massive donor star in a few hundred million years, any presence of persistently bright LMXBs in dSphs, which are dominated by very old stellar populations (>10 Gyr), is not consistent with stellar evolution models (Maccarone et al. 2005). Recent observational studies suggest the presence of candidates for LMXBs in some dwarf galaxies (e.g. Maccarone et al. 2005; Saeedi et al. 2016). However, these few candidates cannot be considered as the main population of the X-ray sources that belong to the dSphs. In the study of the XLF of the Draco dSph, Saeedi et al. (2016) showed that the population of hard X-ray sources $(2.0-10.0 \mathrm{keV})$ in the field of the galaxy does not exceed the population of background sources (background galaxies, AGNs). However, the XLF at 0.5-2.0 keV showed that there might be a population of soft X-ray sources with luminosities $\lesssim 10^{34} \mathrm{erg} \mathrm{s}^{-1}$. This result was consistent with 
the findings of Ramsay \& Wu (2006), who reported that there is a fraction of unclassified soft X-ray sources in Carina and Sagittarius dSphs. An investigation of the nature of the soft lowluminosity X-ray sources in the field of the Draco dSph is the aim of this work.

Low-luminosity X-ray point sources can either be transient LMXBs observed during the low-luminosity state, or accreting white dwarfs (AWDs; Tauris \& van den Heuvel 2006). Since the main population of dSphs is late-type stars (e.g. Tolstoy et al. 2009), white dwarfs are expected to be the main population of compact objects in dSphs and a higher number of AWDs is expected than XRBs. AWDs can be observed in Xrays, UV, optical, and infrared. White dwarfs with a red giant star form binary systems called symbiotic stars, which are considered to be likely candidates for type Ia supernova progenitors (e.g. Kenyon et al. 1993). Steady or quasi-steady burning of accreted matter on the surface of the white dwarf makes the system a super-soft X-ray source (SSS), whereas in wider binary systems, the X-ray emission can have different origins (e.g. Luna et al. 2013). In soft X-ray bands, AWDs have typical luminosities of $10^{31}-10^{34} \mathrm{erg} \mathrm{s}^{-1}$, but in the case of super-soft sources the luminosity range increases to $10^{36}-10^{38} \mathrm{erg} \mathrm{s}^{-1}$ (e.g. Lewin \& van der Klis 2006).

The Draco dSph hosts a known super-soft X-ray source, Draco C 1, studied by Saeedi et al. (2018). In this work we have focussed on finding more candidates of AWDs in the Draco $\mathrm{dSph}$ based on the multi-wavelength study of all unclassified soft $\mathrm{X}$-ray sources.

The first population study of the X-ray sources in the field of Draco $\mathrm{dSph}$ was performed based on five XMMNewton observations obtained in 2009 (Saeedi et al. 2016; Manni et al. 2015; Sonbas et al. 2016). Draco dSph was observed again with 26 deep XMM-Newton observations in 2015. The new observations not only made an extended study of the unclassified sources of the previous catalogues possible, but also revealed many new sources in the field of Draco dSph owing to the increased total exposure time (see Table 1). Saeedi et al. (2016) presented multi-wavelength criteria to distinguish background sources from foreground sources and the members of the Draco dSph. The properties of the optical and infrared counterparts of the X-ray sources were used to identify background sources. This paper presents the classification of fourteen sources detected in the new combined analysis of 2006 and 2015 data, for which a stellar counterpart is confirmed in multiwavelength studies. An updated catalogue of all X-ray sources in the field of Draco dSph is in preparation (Saeedi et al., in prep.).

\section{XMM-Newton, data analysis}

The thirty-one XMM-Newton observations used for our studies are listed in Table 1. The cameras EPIC-pn (Strüder et al. 2001) and EPIC-MOS1, 2 (Turner et al. 2001) were in full-frame mode and the thin filter was used in all observations. Data reduction and source detection were performed using the XMM-Newton Science Analysis System (SAS, V.16.0.0). The event files were screened for time intervals with high background caused by soft proton flares. We extracted single events with PI $>10000$, and PATTERN $=0$ for EPIC-MOS, and PI $>10000$ and PI $<12000$, as well as PATTERN $=0$ for EPIC-pn, and created light curves with time bins of $100 \mathrm{~s}$ to identify background flare intervals. We used a threshold rate of $\leq 0.35$ count s$^{-1}$ for EPIC-MOS and rate $\leq 0.4$ count s$^{-1}$ for EPIC-pn as criteria for the good time intervals. The light curves were also checked visually for good time intervals and possible background flares have been removed.
The final good time interval files were used to filter the event lists. Table 1 lists the final exposure time for each observation and EPIC camera. In this paper we use the number(OBSNo) in Table 1 to specify an XMM-Newton observation instead of the observation ID. Source detection for each observation has been performed using the SAS task edetect-chain in the five standard energy-bands of XMM-Newton B1 $(0.2-0.5 \mathrm{keV})$, B2 (0.5-1.0 keV), B3 (1.0-2.0 keV), B4 (2.0-4.5 keV), B5 (4.5$12.0 \mathrm{keV})$ with minimum detection likelihood of $7^{1}$. Table 2 lists the XMM-Newton ID, position, and position uncertainty for each source. The source numbers $(\mathrm{Src}-\mathrm{No})$ given to each $\mathrm{X}$-ray source in Table 2 are used in the entire paper. The coordinate and the positional error of each source in Table 2 were taken from the observation in which the source was detected with the highest maximum likelihood. The right ascension (RA) and declination(Dec) for each X-ray source was corrected by the offset of the X-ray to the optical position of the known symbiotic star Draco C1 (source No. 5 in this paper) in each observation. We produced a mosaic image using the SAS task edetect-stack from a combination of EPIC images of all observations. Figure 1 shows the three-colour combined image of all observations together in the energy range of $0.2-4.5 \mathrm{keV}$.

\subsection{X-ray timing analysis}

We studied the short and long-term variability of the detected sources of Table 2. To study variability on long timescales, we calculated the weighted flux of the source in each observation from the EPIC cameras, in which the source was detected in the field of view (FOV) in the energy range of $0.2-4.5 \mathrm{keV}$. Band 5(4.5-12 keV) is excluded because the EPIC cameras have a high background contamination and low sensitivity in this energy band. Moreover, except for source No. 1, all the sources studied in this paper emit predominantly X-rays at energies $\lesssim 2 \mathrm{keV}$ (see Figs. 5, 6, and 9). For each source, the count rates have been converted to flux using the energy conversion factor obtained based on the spectral model fitted to the source (see Sect. 2.3). If the spectrum of a source was not available, the energy conversion factor was calculated based on typical models and parameter values for the given source type (see Sects. 4.2.2 and 4.3). For observations in which the position of an X-ray source was in the FOV of the EPIC cameras but the source was not detected, an upper limit was calculated at the position of the source using the sensitivity map in the energy range of $0.2-4.5 \mathrm{keV}$, created using the SAS task esensmap. The long-term X-ray light curves of the sources are shown in Appendix A.

We checked the variability of each source by calculating the ratio of maximum to minimum flux from all observations. The variability (Var) and its significance $(S)$ were calculated using the following formulae:

$\operatorname{Var}=\frac{F_{\max }}{F_{\min }}, \quad S=\frac{F_{\text {max }}-F_{\min }}{\sqrt{E F_{\max }^{2}+E F_{\min }^{2}}}$,

where $F_{\max }$ and $F_{\min }$ are the maximum and minimum X-ray fluxes, and $E F_{\max }$ and $E F_{\min }$ are the errors of the maximum and minimum flux of the source, respectively (Primini et al. 1993). In

\footnotetext{
1 The detection likelihood is calculated by the probability of Poisson random fluctuations of the counts, $L=-\ln (p)$, where $p$ is the probability, which is calculated on the basis of the raw counts of the source and the raw counts of the background maps.
} 
Table 1. XMM-Newton observations of Draco dSph.

\begin{tabular}{|c|c|c|c|c|c|}
\hline OBS-No & OBS-ID & OBS-Date & $\begin{array}{l}\text { EPIC-pn } \\
\text { T.exp }{ }^{(*)} \\
\quad(\mathrm{ks})\end{array}$ & $\begin{array}{c}\text { EPIC-MOS1 } \\
\text { T.exp }^{(*)} \\
(\mathrm{ks})\end{array}$ & $\begin{array}{c}\text { EPIC-MOS2 } \\
\text { T.exp }^{(*)} \\
(\mathrm{ks})\end{array}$ \\
\hline 1 & 0603190101 & 2009-08-04 & 16.2 & 18.8 & 18.8 \\
\hline 2 & 0603190201 & 2009-08-06 & 16.9 & 19.7 & 19.7 \\
\hline 3 & 0603190301 & 2009-08-08 & 9.7 & 13.5 & 13.8 \\
\hline 4 & 0603190401 & 2009-08-20 & 5.8 & 15.8 & 15.9 \\
\hline 5 & 0603190501 & $2009-08-28$ & 16.8 & 19.7 & 19.7 \\
\hline 6 & 0764800101 & 2015-03-18 & 27.2 & 49.3 & 50.1 \\
\hline 7 & 0764800301 & $2015-03-26$ & 25.5 & 56.5 & 57.1 \\
\hline 8 & 0764800401 & $2015-03-28$ & 55.5 & 60.6 & 60.2 \\
\hline 9 & 0764800201 & 2015-04-05 & 26.3 & 41.4 & 40.2 \\
\hline 10 & 0764800501 & 2015-04-07 & 48.2 & 61.7 & 61.7 \\
\hline 11 & 0764800601 & 2015-04-09 & 53.7 & 56.5 & 56.6 \\
\hline 12 & 0764800801 & 2015-04-19 & 28.5 & 48.7 & 50.2 \\
\hline 13 & 0764800901 & 2015-04-25 & 37.2 & 51.7 & 51.7 \\
\hline 14 & 0770180101 & 2015-04-27 & 34.1 & 55.2 & 55.2 \\
\hline 15 & 0770180201 & $2015-05-25$ & 54.7 & 58.7 & 58.7 \\
\hline 16 & 0764800701 & $2015-06-15$ & 56.1 & 57.2 & 57.2 \\
\hline 17 & 0770180401 & 2015-06-18 & 50.9 & 52.7 & 52.7 \\
\hline 18 & 0770180301 & 2015-07-01 & 56.8 & 56.1 & 58.7 \\
\hline 19 & 0770180501 & 2015-07-31 & 51.1 & 54.7 & 54.5 \\
\hline 20 & 0770180701 & $2015-08-22$ & 38.5 & 50.7 & 50.3 \\
\hline 21 & 0770180601 & 2015-09-01 & 38.8 & 67.0 & 66.6 \\
\hline 22 & 0770180801 & 2015-09-03 & 66.6 & 77.4 & 77.8 \\
\hline 23 & 0770190401 & 2015-09-11 & 56.2 & 70.3 & 69.7 \\
\hline 24 & 0770190301 & 2015-09-21 & 20.7 & 43.2 & 40.4 \\
\hline 25 & 0770190101 & 2015-09-23 & 18.3 & 42.8 & 41.9 \\
\hline 26 & 0770190201 & 2015-09-25 & 18.2 & 40.6 & 37.8 \\
\hline 27 & 0770190501 & 2015-10-11 & 32.3 & 44.6 & 44.6 \\
\hline 28 & 0770180901 & $2015-10-13$ & 20.7 & 35.5 & 33.5 \\
\hline 29 & 0770190601 & 2015-10-15 & 7.9 & 23.1 & 24.9 \\
\hline 30 & 0770190701 & $2015-10-17$ & 45.3 & 52.0 & 51.6 \\
\hline 31 & 0770190801 & 2015-10-19 & 32.6 & 48.8 & 48.7 \\
\hline
\end{tabular}

Notes. ${ }^{(*)}$ Exposure time after screening for high background.

Table 2. Catalogue of X-ray sources.

\begin{tabular}{|c|c|c|c|c|}
\hline Src-No & ID & $\begin{array}{c}\text { RA } \\
(\mathrm{J} 2000) \\
\end{array}$ & $\begin{array}{c}\text { Dec } \\
(\mathrm{J} 2000)\end{array}$ & $\begin{array}{r}\mathrm{r} 1 \sigma \\
\left({ }^{\prime \prime}\right) \\
\end{array}$ \\
\hline 1 & XMMUJ171919.8+575943 ${ }^{(*)}$ & 171919.80 & +575943.8 & 0.65 \\
\hline 2 & XMMUJ171920.6+575120 & 171920.61 & +575120.2 & 0.95 \\
\hline 3 & XMMUJ171927.1+580035 ${ }^{(*)}$ & 171927.24 & +580036.2 & 1.30 \\
\hline 4 & XMMUJ171954.1+574244 & 171954.18 & +574244.0 & 1.21 \\
\hline 5 & XMMUJ171957.6+575005 ${ }^{(*)}$ & 171957.65 & +575005.5 & 0.42 \\
\hline 6 & XMMUJ172005.6+575759 ${ }^{(*)}$ & 172005.62 & +575759.2 & 1.45 \\
\hline 7 & XMMUJ172010.2+574823 & 172010.27 & +574823.1 & 1.20 \\
\hline 8 & XMMUJ172013.3+575051 ${ }^{(*)}$ & 172013.39 & +575051.6 & 0.99 \\
\hline 9 & XMMUJ172017.9+575105 ${ }^{(*)}$ & 172017.99 & +575105.7 & 0.48 \\
\hline 10 & XMMUJ172053.8+580044 ${ }^{(*)}$ & 172053.89 & +580044.6 & 0.80 \\
\hline 11 & XMMUJ172104.8+575333 ${ }^{(*)}$ & 172104.79 & +575333.5 & 0.44 \\
\hline 12 & XMMUJ172113.6+580610 & 172113.67 & +5806 10.1 & 1.26 \\
\hline 13 & XMMUJ172131.2+580451 & 172131.20 & +580451.6 & 1.46 \\
\hline 14 & XMMUJ172139.6+575506 & 172139.67 & +575506.9 & 1.54 \\
\hline 15 & XMMUJ172151.4+575700 ${ }^{(*)}$ & 172151.48 & +575700.9 & 1.76 \\
\hline
\end{tabular}

Notes. ${ }^{(*)}$ These sources are also listed in the enhanced $3 \mathrm{XMM}$ catalogue (3XMMe, Rosen 2016).

sources with upper limits lower than the minimum flux, the lowest upper limit was taken to calculate the variability. A source was considered variable if the significance $S$ of the variability factor was greater than three.

We also searched for short-term variability with the following two-steps method: First, for each source we identified observations for which the flux was $>3 \sigma$ above the minimum flux measurement for this source. Here, $\sigma$ is the standard deviation of the flux built from all observations, and the minimum flux is either the lowest detected flux or the lowest upper limit. For all individual observations fullfilling this $3 \sigma$ criterion we examined the background-subtracted lightcurve using the same approach, in other words, we measured the standard deviation of the count rate taking into account all bins in the shortterm light curve and identified the bin with the lowest count rate. All bins with count rate $>3 \sigma$ above this minimum were flagged as variable. The results of this analysis are presented in Sect. 4.

To search for possible periodicity of the sources, we extracted the barycentric corrected event files of each source from the observation, where it had the highest number of counts. We applied the $Z_{1}^{2}$ analysis to the arrival photons $(0.2-12 \mathrm{keV})$ of each source (Buccheri et al. 1983, 1988). We searched for periodic signals in the range of $0.146-10^{4} \mathrm{~s}(0.146$ is the Nyquist limit based on the time resolution of EPIC-pn in full-frame mode, while $10^{4} \mathrm{~s}$ is of the order of the duration of the observation). No 


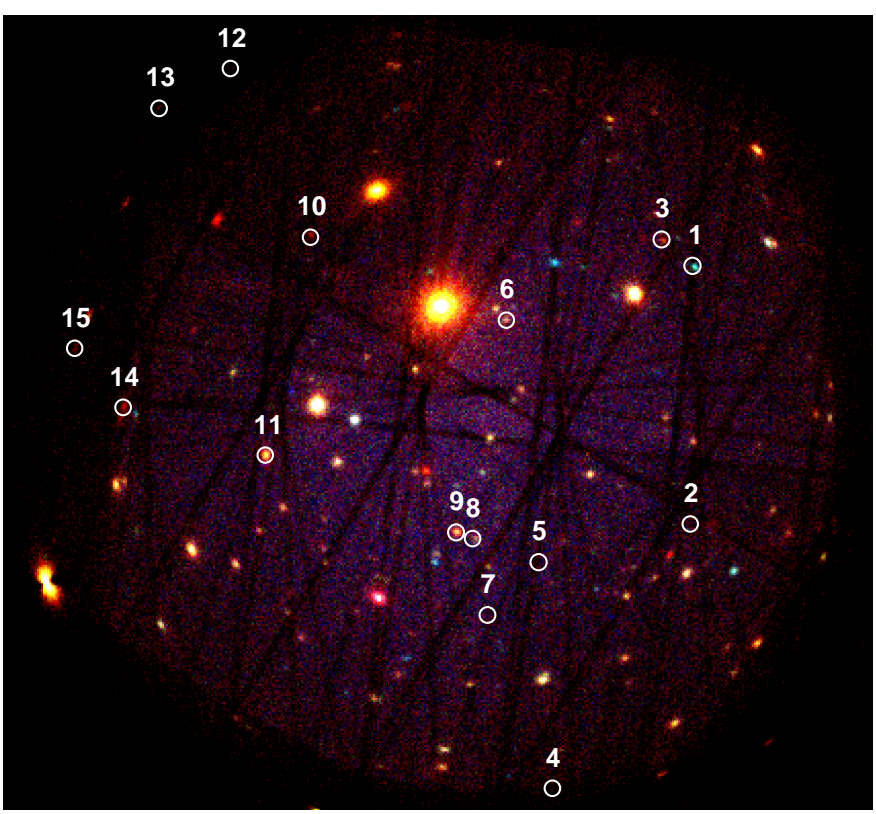

Fig. 1. Combined X-ray image of thirty-one XMM-Newton observations in the field of Draco dSph in the energy band of 0.2-4.5 keV. Sources studied in this work are marked with source numbers from the catalogue in Table 2.

periodic variability was found for any of the sources. Following the method described in Brazier (1994), we found a 90\% upper limit of the pulsed fraction (the pulsed fraction is defined as the semi-amplitude of the modulation divided by the mean source magnitude) of $\approx 85 \%$ in the period range of $0.146-10^{2} \mathrm{~s}$ and $\approx 60 \%$ in the period range of $100-1000 \mathrm{~s}$. The pulsed fractions of accreting pulsars and magnetic white dwarfs can be lower than the aforementioned upper limits (see e.g. Bildsten et al. 1997; Kennea et al. 2009, and references therein), suggesting that these observations are not sensitive enough to set a stringent upper limit on the pulsed fractions of the sources studied in this work.

\subsection{Optical and UV timing analysis}

We reduced the data from the Optical Monitor (OM) onboard XMM-Newton (Mason et al. 2001) using the SAS (V.16.0.0) task omchain. The sources were observed using the $U$ band optical filter (300-390 nm) and the $U V$ filter, $U V W 1(245-320 \mathrm{~nm})$, of the OM telescope in both series of observations in 2009 and 2015. The XMM-OM telescope provides coverage of the central $17^{\prime}$ square region of the EPIC FOV. Therefore, optical light curves are only available for a fraction of the X-ray sources. For observations in which the optical counterpart of the source was located in the FOV of the OM camera, but not detected, the magnitude of the upper limit was calculated using the following formula considering the zero-points of $18.24 \mathrm{mag}$, and $17.37 \mathrm{mag}$ for the $U$ and the $U V W 1$ band, respectively (Mason et al. 2001).

$m=-2.5 \times \log _{10}(\mathrm{DN} / \mathrm{EXPTIME})+$ ZEROPOINT,

where DN is the background number of counts and EXPTIME is the exposure time of the observation (Pogson 1856). The longterm optical and UV light curves of all sources are shown in Appendix A. In addition, We applied the Lomb-Scargle technique for the unevenly sampled time series (Scargle 1982) to search for the possible periodicity in optcial and UV data of the sources. We find no significant periodicity.

\subsection{Spectral analysis}

For sources that have been frequently detected over 31 observations, we obtain a single spectrum with high statistics by merging the spectra of all observations, in which the source was detected. We excluded the spectrum of observations, in which significant flare activity was observed in the light curve of the source according to the analysis described in Sect. 2.1. The source spectrum, background spectrum, ancillary response, and response matrix files of all available observations were combined separately for each EPIC camera, using the SAS task epicspeccombine. In most of the cases, we only used the EPIC-pn data because of their higher statistics compared to EPIC-MOS data. We used EPIC-MOS data in addition to EPIC-pn for sources that have a number of detections in EPICMOS comparable with the EPIC-pn. In fact, excepting source No. 9, the detection in the FOV of EPIC-pn was more frequent than for EPIC-MOS. The data of the combined spectrum has been grouped to a minimum of 20 counts per bin. We used XSPEC (Ver. 12.10.0) to fit the spectra. The models are fitted using the $\chi^{2}$ statistics.

\section{Multi-wavelength studies of counterparts}

In the following, we discuss the multi-wavelength photometry used to uncover the stellar nature of our sources.

\subsection{Optical counterparts of the sources}

The most recent optical survey in the field of Draco dSph is the 9th released data of the Sloan Digital Sky Survey (SDSS9, Ahn et al. 2012). The SDSS9 catalogue contains the magnitudes of the objects in five different energy bands from the near ultraviolet (UV) to the near infrared $(u=3551 \AA, g=4686 \AA, r=$ $6165 \AA, i=7481 \AA, z=8931 \AA$ ). Table B. 1 presents the magnitudes in different bands of SDSS9 for the optical counterparts of the X-ray sources. Appendix C.1 shows the images of the optical counterparts of the X-ray sources taken from SDSS92 ${ }^{2}$. The colour-magnitude diagram of the optical counterparts was plotted using the magnitudes of the $g$ and $r$ bands (Fig. 2). We also plot the $\operatorname{logarithmic} X$-ray to optical flux ratio $\log \left(\frac{F_{\mathrm{X}}}{F_{\mathrm{opt}}}\right)$, versus the X-ray flux (Fig. 3). The flux ratio $\log \left(\frac{F_{\mathrm{X}}}{F_{\mathrm{opt}}}\right)$ was calculated using the modified equation of Maccacaro et al. (1988) in SDSS optical bands (Saeedi et al. 2016):

$\log \left(\frac{F_{\mathrm{X}}}{F_{\text {opt }}}\right)=\log _{10}\left(F_{\mathrm{X}}\right)+\frac{g+r}{2 \times 2.5}+5.37$,

where $F_{\mathrm{X}}$ is the X-ray flux and $g$ and $r$ are the SDSS magnitudes of the optical counterpart associated with the X-ray source. Fig. 3 shows that all sources have $\log \left(\frac{F_{X}}{F_{\text {opt }}}\right)<0$, typical for stars.

For the $u, g, r, i$, and $z$ bands, the Galactic extinction of 0.12 , $0.09,0.06,0.04$, and $0.03 \mathrm{mag}$ is used, respectively, in the direction of the Draco dSph (Schlafly \& Finkbeiner 2011).

\subsection{Infrared counterparts of the sources}

We also searched for mid-infrared counterparts in the WISE All-Sky Data in four energy bands $(3.4,4.6,12$, and $22 \mu \mathrm{m}$, called $W 1, W 2, W 3$, and $W 4$, respectively; Cutri 2012, ) and nearinfrared counterparts in the 2MASS All-Sky Catalogue of Point

\footnotetext{
2 https://dr9.sdss.org/fields
} 


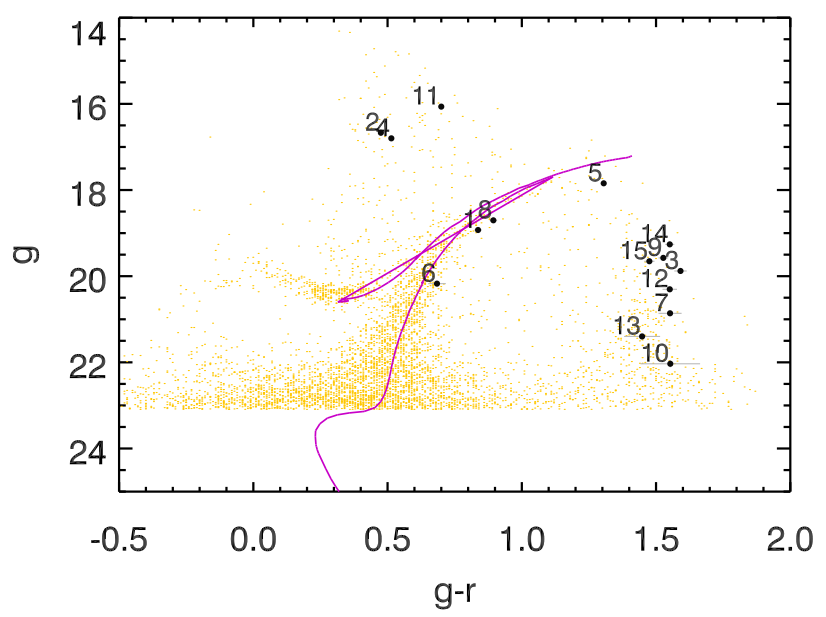

Fig. 2. Colour-magnitude diagram ( $g$ versus $g-r$ ) for the SDSS9 optical counterparts of the X-ray sources (black data points with source No. as labels). Orange dots represent the SDSS7 members ( $g<23 \mathrm{mag}$ ) of the Draco dSph as classified by Rave et al. (2003). The red line is the stellar isochrone for the age of $10 \mathrm{Gyrs}$ and metallicity of 0.0004 solar metallicity of the Draco dSph according to Girardi et al. (2004).

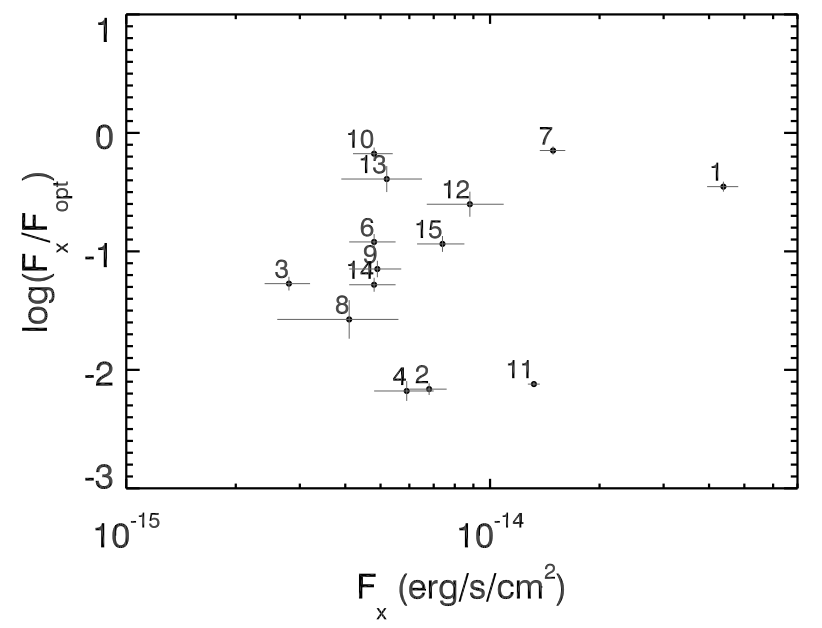

Fig. 3. Logarithmic $\mathrm{X}$-ray to optical flux ratio $\log \left(\frac{F_{\mathrm{X}}}{F_{\mathrm{opt}}}\right)$ versus $F_{\mathrm{X}}$. $\log \left(\frac{F_{\mathrm{X}}}{F_{\mathrm{opt}}}\right)$ is calculated using Eq. (3).

Sources in the $J, H, K$ bands (Cutri et al. 2003). Table B.2 lists the WISE and 2MASS magnitudes of counterparts of the X-ray sources. For the $J, H$, and $K$ bands the Galactic extinction in the direction of the Draco dSph of 0.02, 0.01, 0.01 mag, respectively, are taken into account (Schlegel et al. 1998).

\subsection{Results of multi-wavelength studies}

Photometric studies of the Draco dSph showed that the main population of stars in this dwarf galaxy has already left the main sequence and is on the red giant branch (e.g. Piatek et al. 2001; Bellazzini et al. 2002; Rave et al. 2003; Ségall et al. 2007). The orange dots in Fig. 2 show the main population of stars in the Draco dSph classified by Rave et al. (2003).

In colour-magnitude diagrams, the optical and infrared counterparts of X-ray sources, which are background objects (galaxies and AGNs) are located in different regions compared to the foreground objects and members of Draco dSph. According to Saeedi et al. (2016) the optical and infrared counterparts of non-background sources satisfy the conditions of $\log \left(\frac{F_{\mathrm{X}}}{F_{\mathrm{opt}}}\right)<$

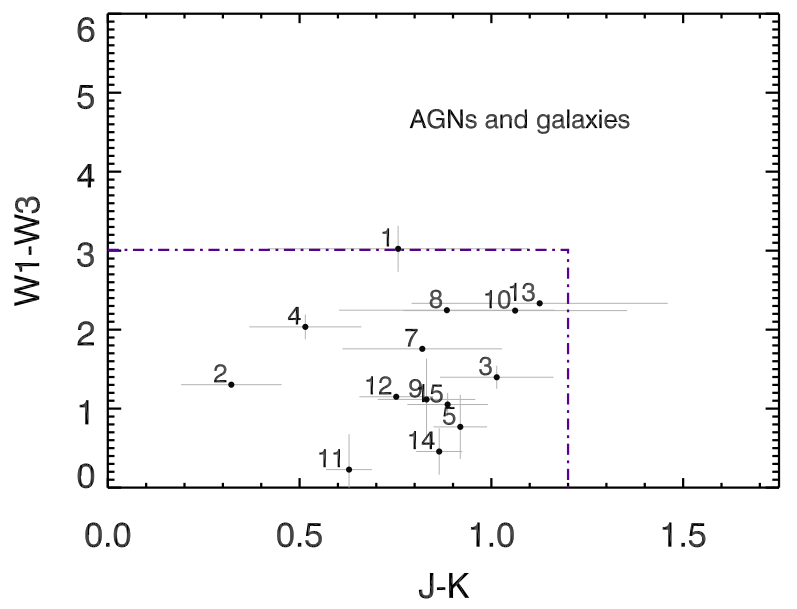

Fig. 4. Colour-colour diagram of mid-infrared WISE $W 1(3.4 \mu \mathrm{m})-$ $W 3(12 \mu \mathrm{m})$ colour index versus near-infrared 2MASS $J-K$ colour index for the counterparts of X-ray sources in the field of the Draco $\mathrm{dSph}$ detected by XMM-Newton. Dash-dotted lines separate the regions, which are occupied by the counterparts of background sources from that of the others (Fig. 3, Saeedi et al. 2016).

$0.0, J-K<1.2$, and $W 1-W 3<3.0$. All the optical and infrared counterparts associated with the X-ray sources, which are studied in this work fulfil these criteria for non-background sources. The colour-colour diagrams of WISE $(W 1-W 3, W 3)$ and 2MASS $(J-K, K)$ show that none of the counterparts is found in the region, where background sources are located (see our Fig. 4, and Fig. 3 of Saeedi et al. 2016). Also, all these X-ray sources have $\log \left(\frac{F_{\mathrm{X}}}{F_{\mathrm{opt}}}\right)<0.0$.

These criteria, which separate the X-ray sources associated with stars from those associated with galaxies and AGNs, are consistent with other photometric classification criteria. Abraham et al. (2012) perform a photometric study to distinguish the sources detected in the SDSS into stars, galaxies, and quasars. They show that stars have very different $u-g$ and $g-r$ colours than quasars. The optical counterparts of all X-ray sources of this work have $u-g>1.5$ and $g-r>1.0$. According to Abraham et al. (2012), they can be classified as stars.

The optical colour-magnitude diagram (Fig. 2) shows that the optical counterparts of some X-ray sources are located at the end of the tail of the red-giant branch of the Draco dSph (sources No. 1, 5, 6, 8) and can be identified as members of the Draco dSph (Sect. 4.1). Optical counterparts associated with sources No. 3, 7, 9, 10, 12, 13, 14, and 15 appear redder and are Galactic M dwarfs (see Sect. 4.2). The optical counterparts of sources Nos. 2, 4, and 11 are located above the red giant branch and seem to be hotter Galactic stars than M dwarfs (Sect. 4.3).

\section{Discussion}

\subsection{Symbiotic stars in the Draco dSph}

The colour-magnitude diagram in Fig. 2 shows that the optical counterparts of the four sources, Nos. 1, 5, 6, and 8 are located on the red-giant branch of Draco dSph. These four sources have also been classified as members of the Draco dSph in other surveys. According to the infrared photometric analysis, symbiotic stars are distinguished into two main groups of either cool star (S-type) with a typical infrared colour of the red giant branch, or as a star with a significant contribution of warm dust (D-type) typical for the asymptotic giant branch (Corradi et al. 2008). The 
Table 3. Best-fit parameters of the X-ray spectrum of source No. 1 for three different models.

\begin{tabular}{|c|c|c|}
\hline \multicolumn{3}{|c|}{ tbabs $\times(\text { apec })^{(*)}$} \\
\hline$N_{\mathrm{H}}$ & $10^{22} \mathrm{~cm}^{-2}$ & $0.83_{-0.11}^{+0.12}$ \\
\hline$k T$ & $\mathrm{keV}$ & $9.57_{-2.22}^{+4.28}$ \\
\hline Norm. & & $\left(2.66_{-0.17}^{+0.18}\right) \times 10^{-5}$ \\
\hline$\chi^{2}$ (d.o.f.) & & $1.05(99)$ \\
\hline$F_{\mathrm{X}}^{(\star)}$ & $\operatorname{erg~s}{ }^{-1} \mathrm{~cm}^{-2}$ & $\left(4.20_{-0.25}^{+0.26}\right) \times 10^{-14}$ \\
\hline \multicolumn{3}{|c|}{ tbabs $\times(\text { mkcflow })^{(*)}$} \\
\hline$N_{\mathrm{H}}$ & $10^{22} \mathrm{~cm}^{-2}$ & $0.98_{-0.16}^{+0.16}$ \\
\hline$k T_{\min }$ & $\mathrm{keV}$ & 0.08 frozen \\
\hline$k T_{\max }$ & $\mathrm{keV}$ & $31.19_{-13.42}^{+36.38}$ \\
\hline Norm. & & $\left(8.67_{-3.83}^{+5.15}\right) \times 10^{-9}$ \\
\hline$\chi^{2}$ (d.o.f.) & & $1.05(99)$ \\
\hline$F_{\mathrm{X}}^{(\star)}$ & $\operatorname{erg~s}^{-1} \mathrm{~cm}^{-2}$ & $\left(4.46_{-0.29}^{+0.30}\right) \times 10^{-14}$ \\
\hline \multicolumn{3}{|c|}{ tbabs $\times($ power-law) } \\
\hline$N_{\mathrm{H}}$ & $10^{22} \mathrm{~cm}^{-2}$ & $0.92_{-0.15}^{+0.17}$ \\
\hline PhoIndex & & $1.62_{-0.16}^{+0.17}$ \\
\hline Norm. & & $\left(7.73_{-1.43}^{+1.80}\right) \times 10^{-6}$ \\
\hline$\chi^{2}$ (d.o.f.) & & $1.10(99)$ \\
\hline$F_{\mathrm{X}}^{(\star)}$ & $\mathrm{erg} \mathrm{s}^{-1} \mathrm{~cm}^{-2}$ & $\left(4.33_{-0.27}^{+0.29}\right) \times 10^{-14}$ \\
\hline
\end{tabular}

Notes. Errors are at the $90 \%$ confidence level. ${ }^{(\star)}$ The absorbed flux is calculated in the energy range of $0.7-8.0 \mathrm{keV} .{ }^{(*)}$ The abundances of the apec and mkcflow models are fixed to the Solar abundances of 1.0.

infrared counterparts of symbiotic stars of the Draco dSph satisfy the conditions of $J-H<1.0, H-K_{s}<0.5, H-W 2<1.0$, and $J-W 1<2$ (see Table B.2), which make them candidates for S-type symbiotic stars (Akras et al. 2019; Corradi et al. 2008). The properties of these systems, which we identify as symbiotic systems in Draco dSph are discussed in the following.

Source No. 1 (XMMUJ171919.8+575943). In the previous work, based on the five XMM-Newton observations of 2009, no optical and infrared counterpart was found for the source. Only a radio counterpart was found and the source was classified as an AGN candidate (Source No. 12, Saeedi et al. 2016). The longer exposure times of the XMM-Newton observations in 2015 yielded a more accurate position of the X-ray source and a bright optical and infrared counterpart was found. Figure 7 shows the $3 \sigma$ error circle of the X-ray source position in observation 17, where source No. 1 had the highest detection maximum likelihood. The optical counterpart of the source is a red giant in Draco dSph. The radial velocity of the source is $-306.59 \mathrm{~km} \mathrm{~s}^{-1}$, which is consistent with that of the Draco dSph members (Kleyna et al. 2002). Kirby et al. (2010) measured an effective temperature of $T_{\text {eff }}=4660 \mathrm{~K}$ and a metallicity of $[\mathrm{Fe} / \mathrm{H}]=-2.12$ for the red giant. The X-ray flux of the source steadily decreased and again increased within about one month in the last observations of 2015 (see Appendix A), which can be a sign of eclipse in the system. The typical orbital period of symbiotic stars is of the order of hundred days (Belczyński et al. 2000). If the observed minimum in the X-ray light curve is related to an eclipse of the white dwarf, its inclination must therefore be very low to explain the short duration of eclipse. On the other hand, UV and optical light curves do not show any evidence for a drop in flux. As a result, the eclipse scenario remains controversial.
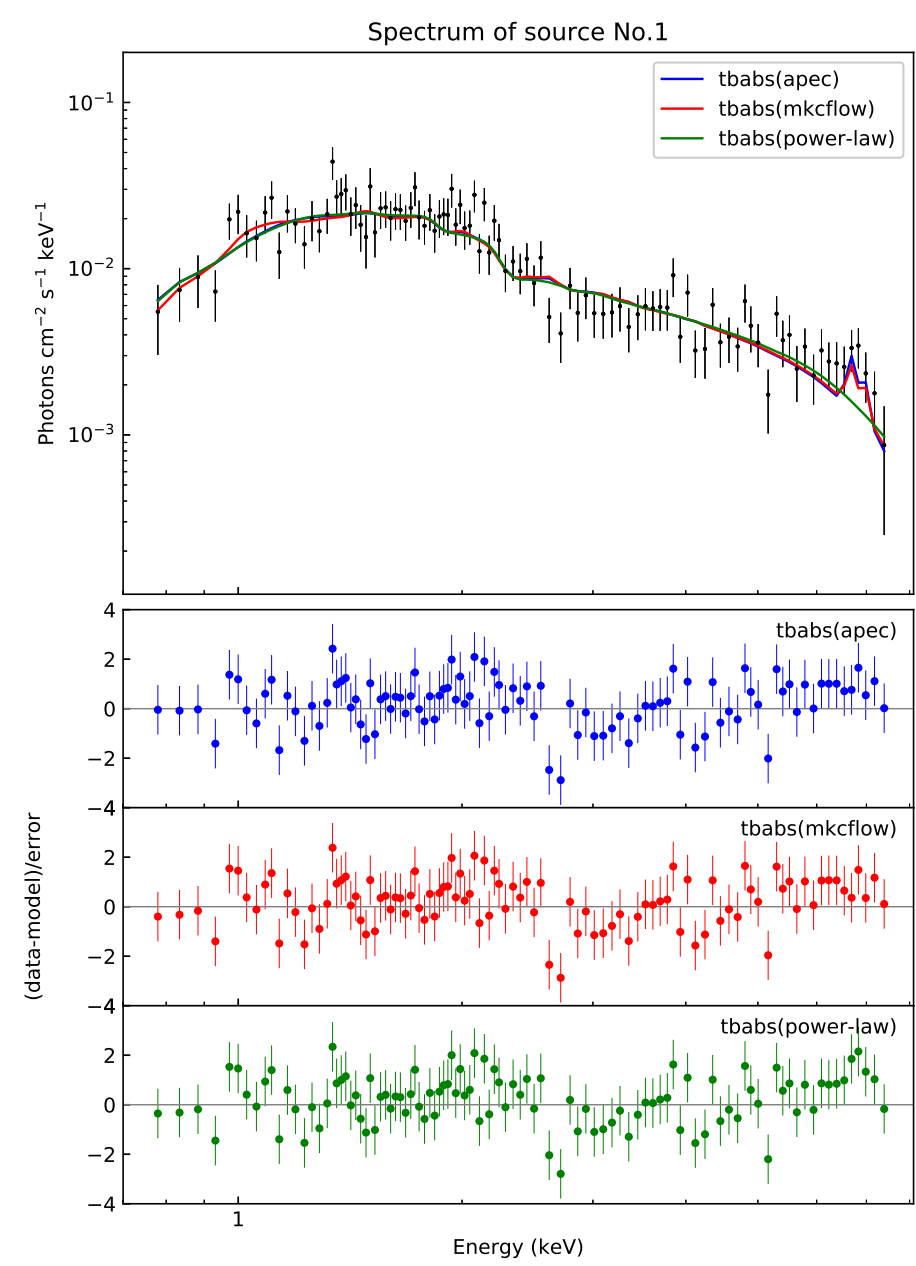

Fig. 5. Combined spectrum of all XMM-Newton observations and bestfit models assuming different types of emission for the source No. 1 , together with the residuals of the fitted models in units of the standard deviation.

The time-averaged X-ray spectrum is fitted very well with an absorbed collisionally-ionized thermal gas model(apec), an absorbed isobaric cooling flow model (mkcflow), and also an absorbed power-law model (see Table 3 and Fig. 5). The parameters of the spectrum of the apec model and mkcflow are very similar to those of symbiotic stars, which show hard $\mathrm{X}$-ray emission and are classified as $\delta$-type symbiotic stars (Muerset et al. 1997; Luna et al. 2013). These symbiotic stars are highly absorbed hard X-ray sources with thermal emission above $2.4 \mathrm{keV}$, which is assumed to originate from the boundary layer of an accretion disc (Luna et al. 2013) similar to Hen 3-461 (Luna et al. 2013), CD-28 3719, 4 Dra (Nuñez et al. 2016), T CrB (Luna et al. 2008), and RT Cru (Luna \& Sokoloski 2007; Ducci et al. 2016). The spectrum is also well fitted with an absorbed power law model. Residuals obtained by subtracting the continuum (Fig. 5, lower panel) show a possible emission feature within the energy range $6.3-7 \mathrm{keV}$, that could be interpreted as noise fluctuation or one (or more) iron line emission. When a Gaussian component is added to the model, the fit is improved by $\Delta \chi^{2} \approx 15.6$. To determine whether the data require this additional component, we used the XSPEC script lrt to perform the likelihood ratio test on $10^{4}$ simulated datasets. We find that the probability that the observed spectrum can be described with an absorbed power law without a Gaussian component is $\sim 0.23 \%$. Therefore, we conclude that the line 
Table 4. Best-fit parameters of the X-ray spectra.

\begin{tabular}{|c|c|c|c|c|c|c|c|c|}
\hline Src-No & Type $^{(\dagger)}$ & Model & $\begin{array}{c}N_{\mathrm{H}} \\
10^{22} \mathrm{~cm}^{-2}\end{array}$ & $\begin{array}{c}k T \\
\mathrm{keV}\end{array}$ & Abundance & $\chi^{2}$ (d.o.f.) & $\begin{array}{c}\text { Absorbed } F_{\mathrm{X}}{ }^{(*)} \\
10^{-15} \mathrm{erg} \mathrm{s}^{-1} \mathrm{~cm}^{-2}\end{array}$ & $\begin{array}{c}L_{X}^{(*)} \\
\operatorname{erg~s}^{-1}\end{array}$ \\
\hline 3 & MD & tbabs $\times($ apec $)$ & $<0.02$ & $0.89_{-0.15}^{+0.20}$ & $<0.1$ & $0.94(26)$ & $3.28_{-0.29}^{+0.34}$ & $1.0 \times 10^{29}$ \\
\hline $5^{(\star)}$ & SS & tbabs $\times$ bb & $0.04_{-0.02}^{+0.02}$ & $0.015_{-0.003}^{+0.003}$ & & $1.35(33)$ & $68.22_{-3.35}^{+3.75}$ & $5.5 \times 10^{34}$ \\
\hline 6 & SS & tbabs $\times$ apec & $<0.03$ & $4.06_{-1.29}^{+2.62}$ & & $0.82(28)$ & $4.75_{-0.73}^{+0.73}$ & $3.9 \times 10^{33}$ \\
\hline 8 & SS & tbabs $\times$ apec & $<0.08$ & $6.2_{-2.3}^{+19.4}$ & & $0.76(40)$ & $4.08_{-1.14}^{+1.53}$ & $3.8 \times 10^{33}$ \\
\hline 9 & MD & tbabs $\times($ apec + apec $)$ & $<0.03$ & $\begin{array}{l}0.21_{-0.03}^{+0.03} \\
0.92_{-0.08}^{0.07}\end{array}$ & $0.33_{-0.15}^{+0.45}$ & $1.03(128)$ & $4.87_{-0.38}^{+0.78}$ & $1.4 \times 10^{29}$ \\
\hline 9 & MD (flare-state) & tbabs $\times($ apec + apec + apec $)$ & $<0.03$ & $\begin{array}{c}\text { Frozen to } 0.21 \\
\text { Frozen to } 0.92 \\
2.84_{0.72}^{+1.61}\end{array}$ & Frozen to 0.33 & $0.95(46)$ & $36.80_{-3.56}^{+3.13}$ & $1.1 \times 10^{30}$ \\
\hline 11 & FG & tbabs $\times($ apec + apec $)$ & $0.05_{-0.01}^{+0.02}$ & $\begin{array}{l}0.27_{-0.04}^{+0.06} \\
1.07_{-0.06}^{+0.06}\end{array}$ & $0.17_{-0.05}^{+0.06}$ & $1.00(174)$ & $13.2_{-0.05}^{+0.05}$ & $1.2 \times 10^{30}$ \\
\hline 14 & MD & tbads $\times($ apec $)$ & $<0.07$ & $0.78_{-0.10}^{+0.13}$ & $<0.1$ & $1.18(46)$ & $5.36_{-0.66}^{+0.65}$ & $1.2 \times 10^{28}$ \\
\hline 15 & MD & tbabs $\times($ apec + apec $)$ & $\begin{array}{c}\text { Frozen to } 0.02 \\
0.12_{-0.08}^{+1.05}\end{array}$ & $\begin{array}{l}0.21_{-0.06}^{+0.11} \\
1.02_{-0.19}^{+0.25}\end{array}$ & $0.14_{-0.11}^{+0.41}$ & $1.66(29)$ & $7.38_{-1.12}^{1.14}$ & $2.3 \times 10^{29}$ \\
\hline
\end{tabular}

Notes. Errors are at the $90 \%$ confidence level. ${ }^{(\dagger)}$ The types of sources are symbiotic stars (SS), Galactic Mdwarfs (MD), and other types of foreground star (FG). ${ }^{(*)}$ Flux and luminosity of all sources are calculated in the energy range of $0.2-5.0 \mathrm{keV}$, except sources No. 1 and No. 8, which are in the energy range of $0.2-10 \mathrm{keV} .{ }^{(\star)}$ Source No. 5 is the super-soft symbiotic white dwarf system Draco C1. The fitted parameters for this source are taken from Saeedi et al. (2018).

significance is marginally larger than $3 \sigma$. The $90 \%$ uncertainties on the line energy parameter $\left(E_{\text {line }}=6.7_{-0.5}^{+2.0} \mathrm{keV}\right)$ does not allow us to determine which emission lines (Fe XXV, Fe XXVI, and Fe XXIII) are responsible for the observed feature.

Assuming a distance of $\sim 82 \mathrm{kpc}$, the X-ray luminosity of the source is $>10^{34} \mathrm{erg} \mathrm{s}^{-1}$ in the energy range of $0.7-8 \mathrm{keV}$. Such a high $L_{X}$, together with the relatively hard X-ray emission of the source is compatible with the emission coming from the boundary layer around a non-magnetic white dwarf (see Ducci et al. 2016; Luna et al. 2008). It is also reminiscent of the typical $\mathrm{X}$-ray emission from magnetic white dwarfs (e.g. Suleimanov et al. 2005). Also, the good fit of power-law model emphasises that the compact object can be a neutron star too. Therefore, the nature of the compact object remains unclear.

Source No. 5 (Draco C1, XMMUJ171957.6+575005). This source is a known super-soft symbiotic star in the Draco dSph detected by ROSAT in X-rays (Allen 1984; Muerset et al. 1997). We recently studied the properties of this source using all available XMM-Newton observations (Saeedi et al. 2018).

Source No.6 (XMMUJ172005.6+575759). The optical counterpart of this source was classified as a red giant in the Draco dSph with an effective temperature of $T_{\text {eff }}=4973 \mathrm{~K}$ and a metallicity of $[\mathrm{Fe} / \mathrm{H}]=-2.28$ (Kirby et al. 2015). The longterm light curve of the source (Appendix A) shows higher X-ray flux in observations 23 and 31 . We checked the short-term light curves of the source in these two observations but the count rate was too low to significantly observe flares or outburst activity. These high X-ray fluxes are not correlated with changes in the UV and optical $U$ magnitudes. The source showed variations in UV and optical $U$-band light curves, but no evidence of periodicity was seen in the light curves. We could fit the X-ray spectrum with an absorbed apec model (see Table 4 and Fig. 5). The system has an $N_{\mathrm{H}}$ similar to the $N_{\mathrm{H}}$ in the direction of the Draco $\mathrm{dSph}$. The statistics of the spectrum are too poor to determine the element abundance of the emitting gas. Therefore, the abundance is left at the default abundance of the apec model (1.0 times the solar values). The soft X-ray spectrum of the source makes it a candidate for a $\beta$-type symbiotic-star. $\beta$-type symbiotic stars have the most part of emission at energies $\lesssim 2 \mathrm{keV}$ (Luna et al. 2013). The X-ray emission is most likely caused by the collision of the wind of the white dwarf with the wind of the red giant (Muerset et al. 1997). However, in this case the system must have been observed during the outburst (Luna et al. 2013). Another model assumes that if the system is observed almost edge-on, the scattering of hard photons in a ionized medium around the white dwarf causes $\beta$-type emission (Wheatley \& Kallman 2006). However, Luna et al. (2013) argue that this model makes it unlikely to detect many symbiotic systems with soft X-ray emission, despite there being plenty of these systems. As an alternative explanation, Luna et al. (2013) suggested that the collision of the wind from the accretion disc with the redgiant wind can cause soft X-ray emission. The high temperature of the source suggests that the X-ray emission comes from the colliding region of fast winds. This source has no counterpart in WISE and 2MASS catalogues.

Source No.8 (XMMUJ172013.3+575051). The optical counterpart of this source was classified as a member of the Draco dSph with a radial velocity of $-291.3 \mathrm{~km} \mathrm{~s}^{-1}$ (Armandroff et al. 1995). The long-term light curve of the source shows that the X-ray fluxes in the observations of 2009 were higher than during the observations in 2015. The observations of 2015 show no significant variation over the 26 observations ( $\operatorname{Var}=1.8$, see Sect. 2.1). The X-ray spectrum of the source is soft, which makes it a candidate for a $\beta$-type symbiotic-star. The spectrum is well fitted with an absorbed apec model (see Table 4 and Fig. 5). Based on available catalogues of symbiotic stars (e.g. Belczyński et al. 2000; Luna et al. 2013), Sources No. 6 and 8 in the Draco $\mathrm{dSph}$ are the first identified extragalactic $\beta$-type symbiotic stars.

\subsection{Galactic Mdwarfs in the field of Draco dSph}

\subsubsection{Optical properties of Mdwarfs}

The counterparts of sources No.3, 7, 9, 10, 12, 13, 14, and 15 have infrared magnitudes and colours consistent with those expected from symbiotic systems in the Draco dSph (see Fig. 4), while in the optical colour-magnitude diagram (Fig. 2) they are 
Table 5. Optical and X-ray properties of classified Galactic M dwarfs in the filed of the Draco dSph.

\begin{tabular}{lcccccccccc}
\hline \hline Src-No & $r-i$ & $i-z$ & $z-J$ & Spectral type & $M_{i}$ & $d_{\text {phot }}(\mathrm{pc})$ & $d_{\text {Gaia }}(\mathrm{pc})$ & $M_{\text {bol }}$ & $L_{\mathrm{X}}\left(\mathrm{erg} \mathrm{s}^{-1}\right)$ & $\log \left(\frac{L_{\mathrm{X}}}{L_{\text {bol }}}\right)$ \\
\hline 3 & $0.92 \pm 0.01$ & $0.52 \pm 0.02$ & $1.21 \pm 0.08$ & M2 & $8.8 \pm 0.1$ & 506 & - & 8.6 & $8.5 \times 10^{28}$ & -2.9 \\
7 & $1.33 \pm 0.02$ & $0.70 \pm 0.02$ & $1.38 \pm 0.10$ & M4 & $9.34 \pm 0.3$ & 520 & $366 \pm 23$ & 9.5 & $3.2 \times 10^{29}$ & -2.1 \\
9 & $1.15 \pm 0.01$ & $0.64 \pm 0.01$ & $1.28 \pm 0.06$ & M3 & $8.8 \pm 0.3$ & 413 & $497 \pm 20$ & 8.2 & $1.4 \times 10^{29}$ & -3.0 \\
10 & $1.74 \pm 0.04$ & $0.98 \pm 0.03$ & $1.59 \pm 0.13$ & M5 & $11.7 \pm 0.4$ & 242 & $657 \pm 160$ & 9.1 & $2.5 \times 10^{29}$ & -2.5 \\
12 & $1.40 \pm 0.02$ & $0.78 \pm 0.02$ & $1.51 \pm 0.05$ & M4 & $10.0 \pm 0.3$ & 351 & $446 \pm 28$ & 8.7 & $2.0 \times 10^{29}$ & -2.7 \\
13 & $1.14 \pm 0.04$ & $0.64 \pm 0.04$ & $1.32 \pm 0.19$ & M3 & $9.2 \pm 0.5$ & 820 & - & 9.7 & $3.0 \times 10^{29}$ & -2.4 \\
14 & $1.45 \pm 0.01$ & $0.80 \pm 0.01$ & $1.44 \pm 0.04$ & M4-M & $10.2 \pm 0.3$ & 158 & $140 \pm 2$ & 9.7 & $1.2 \times 10^{28}$ & -3.3 \\
15 & $1.15 \pm 0.01$ & $0.62 \pm 0.01$ & $1.37 \pm 0.06$ & M3 & $9.1 \pm 0.1$ & 375 & $510 \pm 70$ & 8.3 & $2.3 \times 10^{29}$ & -2.8 \\
\hline
\end{tabular}

Notes. The spectral types of the Mdwarfs are identified based on the optical and infrared colours (West et al. 2011). The table also presents the absolute magnitude $\left(M_{i}\right)$, bolometric magnitude $\left(M_{\mathrm{bol}}\right)$, photometric distance $\left(d_{\text {phot }}\right)$, Gaia DR2 distance $\left(d_{\text {Gaia }}\right)$, X-ray luminosity $\left(L_{\mathrm{X}}\right)$ in the energy range of $0.2-5.0 \mathrm{keV}$, and X-ray to bolometric luminosity, $\log \left(\frac{L_{X}}{L_{\mathrm{bol}}}\right)$, of M dwarfs.

separated from the red giant branch of the Draco dSph. All of them were classified as stars (see Sect. 3.3). However, their peculiar position in the colour-magnitude diagram of Fig. 2 required further studies to determine their spectral type. We find that all these sources satisfy the conditions for M dwarfs for the SDSS colours $r-i<0.42$ and $i-z<0.24$ (West et al. 2005). We also estimated the spectral type of the counterparts using the characterization of Mdwarfs given by West et al. (2011) through the observed colours $r-i, i-z, z-J$, and $J-H$. Moreover, based on the optical SDSS colours of the M dwarfs the absolute magnitude of the M dwarfs in SDSS $i$ band using $i-z$ colours are estimated (see Table 5). The photometric distances derived this way show that all sources are Galactic M dwarfs located at distances of few hundred parsecs.

The second release of Gaia (DR2, Gaia Collaboration 2018) provides distances based on parallax measurements. We examined whether the differences between the photometric and astrometric distances (of up to a factor of two; see Table 5) might be due to problematic solutions in Gaia DR2. To this end, we used the filters defined by Arenou et al. (2018) and Lindegren et al. (2018, Appendix C, Eqs. C-1 and C-2) and additional quality indicators of the solutions (ASTROMETRIC_EXCESS_NOISE, ASTROMETRIC_GOF_AL). The only criterion of this quality check that is not fullfilled by all stars is the ASTROMETRIC_EXCESS_NOISE. However, the values of the ASTROMETRIC_EXCESS_NOISE are small for all objects, and similar values have been deemed acceptable in other studies (Lindegren et al. 2018). Therefore, we decided to consider the Gaia DR2 distances as validated and we used them for the calculation of the luminosities. The bolometric luminosity of each $\mathrm{M}$ dwarf was calculated using the bolometric correction of the $i$ band (Mann et al. 2015). We also estimated the logarithmic ratio of X-ray to bolometric $\operatorname{luminosity~} \log \left(\frac{L_{\mathrm{X}}}{L_{\mathrm{bol}}}\right)$ for each source. $\mathrm{X}$-ray luminosity and $\mathrm{X}$-ray to bolometric luminosity ratio are listed in Table 5.

\subsubsection{X-ray analysis of Mdwarfs}

Mdwarfs are also known as flare stars due to their frequent brightness increases resulting from magnetic reconnection events. The timescale of stellar X-ray flares is hours to days (e.g. Güdel 2004). As the typical duration of the observations analysed here is $\sim 30-50 \mathrm{ks}$, we expect to identify the typical variability of flares, if present, in the lightcurve of individual observations. In fact, with the procedure described in Sect. 2.1 we have identified flares for sources No. 3, 9, 10, 14, and 15. The poor count statistics imply that most of these events are charaterized by a single bin in the short-term lightcurve. Therefore, we
Table 6. Flux variation of M dwarfs in flaring states.

\begin{tabular}{llll}
\hline \hline Source No. & OBS-No. & $\begin{array}{l}F_{\min } \\
\left(\mathrm{erg} \mathrm{s}^{-1} \mathrm{~cm}^{-2}\right)\end{array}$ & $\begin{array}{l}\Delta F_{\min } \\
\left(\mathrm{erg} \mathrm{s}^{-1} \mathrm{~cm}^{-2}\right)\end{array}$ \\
\hline 3 & 11 & $(1.5 \pm 0.4) \times 10^{-15}$ & $3.2 \times 10^{-15}$ \\
9 & 3 & $(5.8 \pm 1.5) \times 10^{-15}$ & $3.8 \times 10^{-14}$ \\
9 & 4 & $(5.8 \pm 1.5) \times 10^{-15}$ & $1.8 \times 10^{-13}$ \\
9 & 5 & $(5.8 \pm 1.5) \times 10^{-15}$ & $1.1 \times 10^{-14}$ \\
9 & 20 & $(5.8 \pm 1.5) \times 10^{-15}$ & $7.0 \times 10^{-14}$ \\
9 & 22 & $(5.8 \pm 1.5) \times 10^{-15}$ & $9.4 \times 10^{-15}$ \\
10 & 17 & $(1.5 \pm 0.4) \times 10^{-15}$ & $3.7 \times 10^{-15}$ \\
14 & 19 & $(2.2 \pm 0.6) \times 10^{-15}$ & $4.0 \times 10^{-15}$ \\
15 & 14 & $(2.0 \pm 0.7) \times 10^{-15}$ & $1.3 \times 10^{-14}$ \\
15 & 30 & $(2.0 \pm 0.7) \times 10^{-15}$ & $1.0 \times 10^{-14}$ \\
\hline
\end{tabular}

cannot examine the morphology of the flares. The exception is a long and bright flare of source No. 9 that we describe in the following. For the other events we list the minimum duration and minimum flux increase in Table 6. Highlight results of timing and spectral analyses are explained in the following:

Source No. 9. This source is classified as the most variable source in the field of the Draco dSph due to its very high flux in observation 4 (source No. 33, Saeedi et al. 2016). In observation 20 the source again showed strong variability. The long exposure time of observation 20 allowed us to study the shortterm X-ray light curve and the X-ray spectrum of the star during this flare. Figure 8 shows the light curve of the source in observations 19,20 , and 21 , which represent the times before, during, and after the strong flare. It seems that the flare started before the beginning of observation 20 and therefore, we can only give a lower limit to the flare duration of $>15 \mathrm{~h}$. The X-ray spectrum of the source in the quiescent state was fitted with two absorbed apec models (see Table 4 and Fig. 6). The spectrum of the source in observation 20, when the source was flaring, required an additional third thermal component. The first two components were fixed to the values previously obtained during the quiescent state. The third apec component is significantly hotter than the temperatures representing the quiescent spectrum. The spectral analysis yields a temperature of $\sim 32 \mathrm{MK}$ and an average X-ray luminosity of $10^{30} \mathrm{erg} \mathrm{s}^{-1}$ (see Table 4), which are typical for Xray flares of cool stars (e.g. Güdel 2004). The $\log \left(\frac{L_{X}}{L_{\text {bol }}}\right)$ in the quiescent state was -3.0 and increased to -2.1 in the strong flare. The long-term light curve in the $U$ band shows evidence for enhanced emission during the X-ray flare (observations 20, Appendix A). 
S. Saeedi et al.: Classification of low luminosity stellar X-ray sources of the Draco dSph
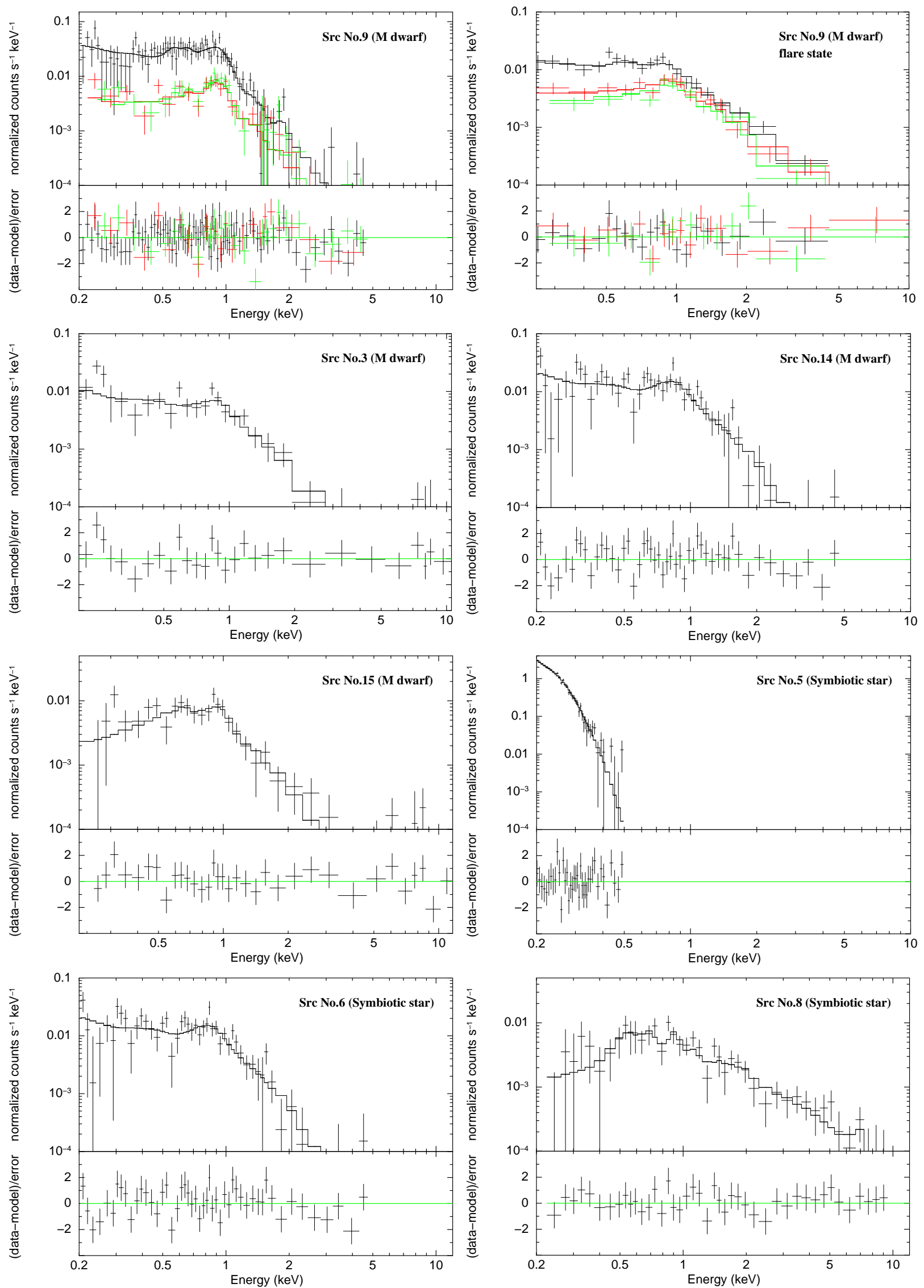

Fig. 6. Combined spectrum of all XMM-Newton observations of sources (M dwarfs and symbiotic stars) in the field of Draco dSph, which have enough statistics for spectral analysis: EPIC-pn (black), EPIC-MOS1 (red), and EPIC-MOS2 (green), together with the residuals in units of the standard deviation. 


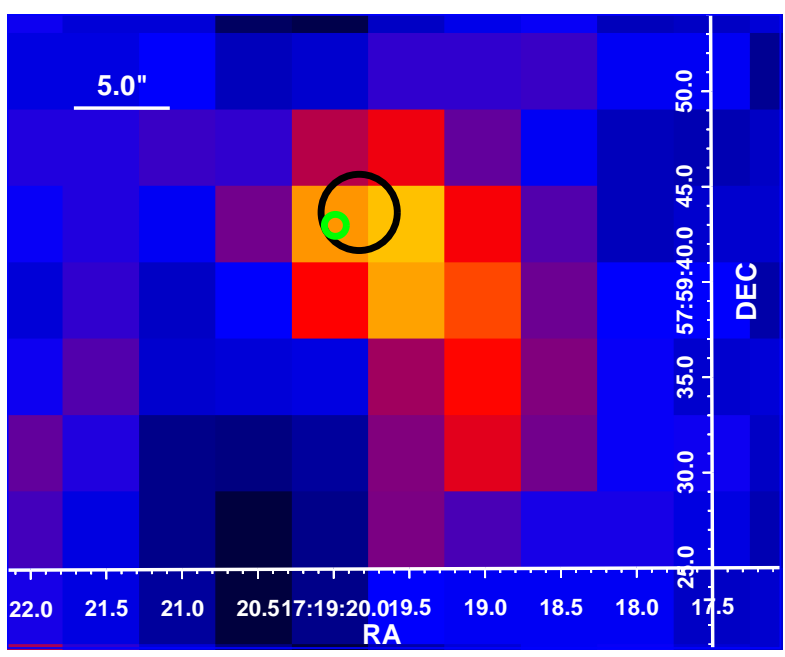

Fig. 7. X-ray mosiac image of Source No. 1 in the energy band $0.2-$ $12.0 \mathrm{keV}$. The black circle shows the X-ray position of the source. The green circle shows the position of the optical counterpart.

Sources No. 3, 14, and 15. We fitted absorbed one or twotemperature apec model to their spectra (Table 4$)$. The best fit temperatures of the sources and their abundance $(<$ with respect to the solar abundance) are very similar to each other. The X-ray luminosity of all these sources, based on the Gaia distances, are presented in Table 4.

Sources No. 7, 10, 12, and 13. We could not fit the spectrum due to the very low statistics of their combined spectra. Therefore, we estimated the flux of these sources assuming a typical model fitted to the spectrum of a Mdwarf using the parameters of Table 4 , which means an absorbed apec model with a Galactic $N_{\mathrm{H}}$ of $2.5 \times 10^{20} \mathrm{~cm}^{-2}$, a temperature of $k T=0.7 \mathrm{keV}$, and a sub-solar abundance of 0.2 . The X-ray luminosity of these sources are presented in Table 5. Source No. 7 has $\log \left(\frac{L_{X}}{L_{\mathrm{bol}}}\right)$ of -2.1 , which is higher than the typical $\log \left(\frac{L_{X}}{L_{\text {bol }}}\right)$ of M dwarfs. The source is detected only twice over all observations. All the upper limit fluxes at the position of the source are lower than the flux of the source measured in observations 6 and 18 (Appendix A). This suggests that source No. 7 was detected only during flare activity. However, the count rates were too low to identify a flare signature in the short-term light curves of observations 6 and 18. Sources No. 10, 12, and 13 have typical X-ray to bolometric luminosity for M dwarfs in the quiescent state.

\subsubsection{X-ray population}

Mdwarfs are the most numerous stars in the Galaxy (Liebert 1994). Their X-ray emission originates from magnetic activity caused by a stellar dynamo (Parker 1975). Stars with spectral types later than $\sim \mathrm{M} 3$ are fully convective, therefore the field generating mechanism is expected to be different from the solar-type $\alpha \Omega$ dynamo. The spectral types we derived for $\mathrm{Mdwarfs}$ from $X M M-N e w t o n$ data in the Draco dSph field suggest that they belong to this category. All of them have high $\log \left(\frac{L_{X}}{L_{\text {bol }}}\right)$ ratios typical for fast-rotating $\mathrm{M}$ dwarfs in the saturation regime, while the full population of $\mathrm{Mdwarfs}$ is characterized by a spread of more than three dex reaching down as low as $\log \left(\frac{L_{\mathrm{X}}}{L_{\mathrm{bol}}}\right) \sim-6$ (Stelzer et al. 2013). Evidently, only the most active M dwarfs in the Draco field are detected with XMM-Newton.

From the space density of M dwarfs presented by Bochanski et al. (2010) based on the SDSS we estimate a total number of

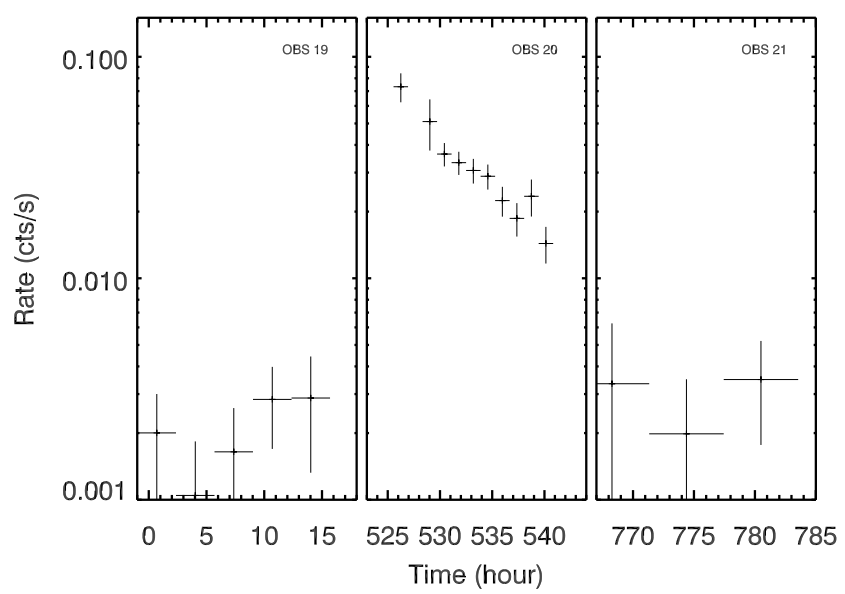

Fig. 8. X-ray light curves of source No.9 in the observations 19, 20 (during the strong flare), and 21. The data is taken from the EPIC-pn camera.

$\sim 480 \mathrm{Mdwarfs}$ in the $0.25 \mathrm{deg}^{-2}$ FOV of XMM-Newton for a volume reaching to a distance of $500 \mathrm{pc}$ (i.e. the average distance of the Mdwarfs detected in this work). This shows that these exceptionally deep X-ray observations have allowed us to detect $\sim 2 \%$ of the Galactic M dwarfs in the surveyed area. This is consistent with the results derived from the X-ray luminosity function of the volume-limited sample of $\mathrm{M}$ dwarfs within $10 \mathrm{pc}$ of the Sun, where only 3 out of 159 stars (i.e. $2 \%$ ) have $L_{X}$ values above the ones observed in our sample, meaning greater than $10^{29} \mathrm{erg} \mathrm{s}^{-1}$ (Stelzer et al. 2013).

\subsection{Other classified Galactic sources in the field of Draco dSph}

Sources No. 2, 4, and 11 have infrared colours and magnitudes similar to the symbiotic stars in the Draco dSph (see Fig. 4). However, their optical apparent magnitude is higher than those of members of the Draco dSph (Fig. 2). The parallax measurements of Gaia DR2 show that the counterparts of these X-ray sources are Galactic objects located at distances of $\sim 1-3 \mathrm{kpc}$. Table 7 shows the distance presented for the classified foreground X-ray sources in the field of Draco dSph in the work of Saeedi et al. (2016) and the three new classified foreground X-ray sources of this work. The filtering conditions of Gaia parallax (see Sect. 4.2.1) confirm that the parallax measurement of all them are acceptable. The distances of these three new foreground sources are larger than the distances of the previous six foreground X-ray sources in the work of Saeedi et al. (2016), which were located at $\sim 100-600 \mathrm{pc}$, consistent with the higher sensitivity for the detection of faint X-ray sources thanks to the increased exposure time available now.

Source No. 2 was detected in X-rays only three times over the observations of 2015. The statistics are too poor to analyse the spectrum of the source. Most source counts were received in the soft energy range of $0.2-2.0 \mathrm{keV}$, therefore we assumed an absorbed apec model with the Galactic $N_{\mathrm{H}}$ of $2.5 \times 10^{22} \mathrm{~cm}^{-2}$ and a temperature of $k T=1.0 \mathrm{keV}$. The minimum and maximum flux of the source is between $(2-7) \times 10^{-15} \mathrm{erg} \mathrm{s}^{-1} \mathrm{~cm}^{-2}$. Thus, assuming a distance of $\sim 2 \mathrm{kpc}$ (see Table 7 ) the X-ray luminosity is $\sim(1-4) \times 10^{30} \mathrm{erg} \mathrm{s}^{-1}$. The flux of the source in observation 30 is significantly higher than in observations 8 and 10, but no flare is observed in the short-term light curve of observation 30 . The $g-r$ colour of $\sim 0.45$ mag suggests a spectral type of $\mathrm{F}$ or $\mathrm{G}$ for 
Table 7. Distance of additional classified foreground stars in the field of the Draco dSph.

\begin{tabular}{|c|c|}
\hline Src-ID & Distance $(\mathrm{pc})$ \\
\hline XMMUJ171925.97+575020.1 ${ }^{(*)}$ & $224 \pm 36$ \\
\hline XMMUJ172021.83+575827.3 $3^{(*)}$ & $95.1 \pm 0.6$ \\
\hline XMMUJ172025.65+575304.4 ${ }^{(*)}$ & $566 \pm 5$ \\
\hline XMMUJ172037.64+580211.9 ${ }^{(*)}$ & $515 \pm 7$ \\
\hline XMMUJ172116.97+580113.6 ${ }^{(*)}$ & $299 \pm 2$ \\
\hline XMMUJ172158.29+574922.5 ${ }^{(*)}$ & $431 \pm 6$ \\
\hline XMMUJ172104.8+575333.5 (No. 11) & $860 \pm 22$ \\
\hline XMMUJ171920.6+575120.2(No. 2) & $2094 \pm 168$ \\
\hline XMMUJ171954.2+574244.0(No. 4) & $2665 \pm 300$ \\
\hline
\end{tabular}

Notes. ${ }^{(*)}$ Foreground sources, which were classified in Saeedi et al. (2016).

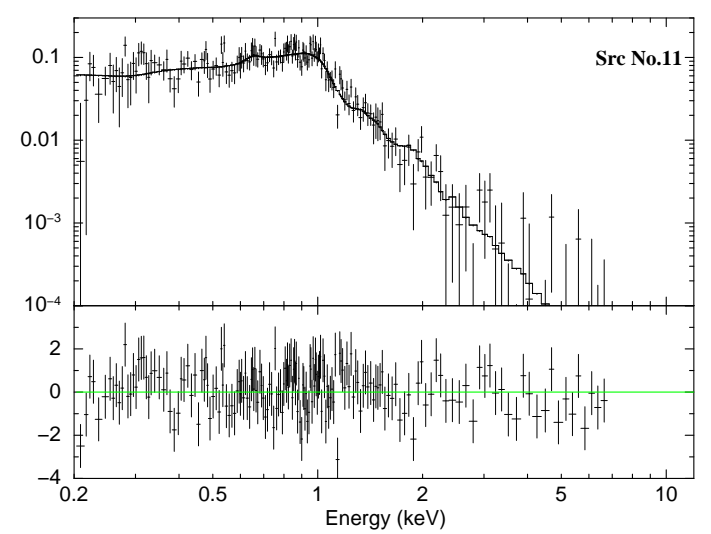

Fig. 9. Combined spectrum of XMM-Newton observations (EPIC-pn) of the foreground star source No. 11, together with the residuals in units of the standard deviation.

the optical counterpart (Yanny et al. 2009). The UV and optical counterpart of the source was always detected by OM if it was inside its FOV.

Source No. 4 has an X-ray detection in four observations during the 2015 campaign (see Appendix A). The statistics were too low for a spectral fit. Therefore, we assumed the same model as for source No. 2 to estimate the flux of the source. Its average is between $(2-6) \times 10^{-15} \mathrm{erg} \mathrm{s}^{-1} \mathrm{~cm}^{-2}$, that is, a luminosity of $\sim(2-5) \times 10^{30} \mathrm{erg} \mathrm{s}^{-1}$ at the distance of $2.6 \mathrm{kpc}$. No variability is detected for this source. The $g-r$ colour of $\sim 0.5$ mag suggests a spectral type of $\mathrm{G}$ for the optical counterpart (Yanny et al. 2009). The source was outside the FOV of the OM telescope.

Source No. 11 had already been detected in five 2009 observations. However, it remained unclassified due to the lack of information on the counterpart. The extended X-ray light curve including the data from year 2015 shows that it is a variable $\mathrm{X}$-ray source. We identify a flare in observation 25 . The timeaveraged X-ray spectrum of the source was well fitted with an absorbed two-temperature apec model (see Table 4 and Fig. 9). The $g-r$ colour of $\sim 0.6$ mag suggests a spectral type of $\mathrm{K}$ for the optical counterpart (Yanny et al. 2009).

Both the luminosity of the three classified foreground sources in this work $\left(>10^{30} \mathrm{erg} \mathrm{s}^{-1}\right)$ and the spectral types of their optical counterparts ( $\mathrm{G}$ or $\mathrm{K}$ type) make them candidates for contact binary systems (e.g. Shaw et al. 1996; Geske et al. 2006). However, more observations in the optical band are necessary to study the light curves and emission lines of these sources.

\section{Summary}

This study presents multi-wavelength criteria to distinguish X-ray sources in the Draco dSph from Galactic stars using deep $X M M-N e w t o n$ observations. We show that all sources with a stellar counterpart have $J-K<1.2$ and $W 1-W 3<3.0$ colours in the infrared. All classified accreting white dwarfs and foreground stars have $\log \left(\frac{F_{\mathrm{X}}}{F_{\text {opt }}}\right)<0.0$. Also, the colour magnitude diagram of optical counterparts of the X-ray sources is a useful key to identify the spectral type of the stellar counterparts. These criteria are specially helpful in the classification of accreting white dwarfs, which have bright optical and infrared counterparts in nearby galaxies.

Based on the above criteria, we classiyi fifteen X-ray sources with stellar counterparts in the field of the Draco dSph. We provid X-ray timing (periodicity and other variability) and spectral analyses for these sources.

We classify three new symbiotic stars in the Draco dSph, in addition to the known super-soft symbiotic star Draco C1 (Saeedi et al. 2018). The X-ray spectra of the three symbiotic stars revealed one $\delta$-type symbiotic star (source No. 1) and two $\beta$-type symbiotic stars (sources No. 6 and No. 8), which are the first classified extragalactic $\beta$-type symbiotic stars. This study shows that white dwarf binaries are the most prominent class of $\mathrm{X}$-ray sources in the field of $\mathrm{dSphs}$ as expected from theoretical studies (see Sect. 1).

Eight sources are classified as Galactic Mdwarfs in the field of the Draco dSph with spectral types between M2-M5. The $\mathrm{X}$-ray luminosity of these M dwarfs range between $10^{28}-3 \times$ $10^{29} \mathrm{erg} \mathrm{s}^{-1}$. We calculate a detection fraction of $\sim 2 \%$ based on estimates of the space density of M dwarfs. The observed X-ray luminosities are consistent with those of the upper $2 \%$ of the $\mathrm{M}$ dwarf X-ray luminosity function of the volume-limited sample of Mdwarfs within a $10 \mathrm{pc}$ distance from the Sun. For five Mdwarfs we observed flaring activity. The X-ray spectrum and light curve of source No. 9 was studied during both the quiescent and the flaring states showing the typical temporal and spectral signatures of flares, that is, exponentially decaying brightness and increased X-ray temperature during the bright state. Finally, we classify three sources as more distant Galactic stars, which are candidates for contact binaries.

This study is part of a new X-ray study of the Draco $\mathrm{dSph}$. Here, we present the study of sources that have a stellar counterpart. The new catalogue, which will contain the classification of all other X-ray sources (i.e. mainly classified background objects, X-ray sources without any counterpart, and $\mathrm{X}$-ray sources with unclassified counterparts) is in preparation (Saeedi et al, in prep.).

Acknowledgements. We thank the anonymous referee for the useful comments that helped us to improve the paper. This research was funded by the Deutsche Forschungsgemeinschaft (DFG) through the Heisenberg research grant SA 2131/4-1. M.S. acknowledges support by the DFG through the Heisenberg professor grants SA 2131/5-1, 12-1. L.D. acknowledges support by the Bundesministerium für Wirtschaft und Technologie and the Deutsches Zentrum für Luft und Raumfahrt through the grant FKZ 50 OG 1602. This study is based on observations obtained with XMM-Newton, an ESA science mission with instruments and contributions directly funded by ESA Member States and NASA. This research has made use of the SIMBAD and VIZIER database, operated at CDS, Strasbourg, France, and of the NASA/IPAC Extragalactic Database (NED), which is operated by the Jet Propulsion Laboratory, California Institute of Technology, under contract with the National Aeronautics and Space Administration. This publication makes use of data products from the Wide-field Infrared Survey Explorer, which is a joint project of the University of California, Los Angeles, and the Jet Propulsion Laboratory/California Institute of Technology, funded by the National Aeronautics and Space Administration. This publication has made use of data products from the Two Micron All Sky Survey, 
which is a joint project of the University of Massachusetts and the Infrared Processing and Analysis Center, funded by the National Aeronautics and Space Administration and the National Science Foundation. Funding for SDSS and SDSS-III has been provided by the Alfred P. Sloan Foundation, the Participating Institutions, the National Science Foundation, and the US Department of Energy Office of Science. The SDSS-III web site is http://www.sdss3.org/ SDSS-III is managed by the Astrophysical Research Consortium for the Participating Institutions of the SDSS-III Collaboration including the University of Arizona, the Brazilian Participation Group, Brookhaven National Laboratory, University of Cambridge, University of Florida, the French Participation Group, the German Participation Group, the Instituto de Astrofisica de Canarias, the Michigan State/Notre Dame/JINA Participation Group, Johns Hopkins University, Lawrence Berkeley National Laboratory, Max Planck Institute for Astrophysics, New Mexico State University, New York University, Ohio State University, Pennsylvania State University, University of Portsmouth, Princeton University, the Spanish Participation Group, University of Tokyo, University of Utah, Vanderbilt University, University of Virginia, University of Washington and Yale University. This research has made use of SAOImage DS9, developed by Smithsonian Astrophysical Observatory.

\section{References}

Abraham, S., Philip, N. S., Kembhavi, A., Wadadekar, Y. G., \& Sinha, R. 2012, MNRAS, 419, 80

Ahn, C. P., Alexandroff, R., Allende Prieto, C., et al. 2012, ApJS, 203, 21

Akras, S., Leal-Ferreira, M. L., Guzman-Ramirez, L., \& Ramos-Larios, G. 2019, MNRAS, 483, 5077

Allen, D. A. 1984, Proc. Astron. Soc. Aust., 5, 369

Aoki, W., Arimoto, N., Sadakane, K., et al. 2009, A\&A, 502, 569

Arenou, F., Luri, X., Babusiaux, C., et al. 2018, A\&A, 616, A17

Armandroff, T. E., Olszewski, E. W., \& Pryor, C. 1995, AJ, 110, 2131

Belczyński, K., Mikołajewska, J., Munari, U., Ivison, R. J., \& Friedjung, M 2000, A\&AS, 146, 407

Bellazzini, M., Ferraro, F. R., Origlia, L., et al. 2002, AJ, 124, 322

Bildsten, L., Chakrabarty, D., Chiu, J., et al. 1997, ApJS, 113, 367

Bochanski, J. J., Hawley, S. L., Covey, K. R., et al. 2010, AJ, 139, 2679

Brazier, K. T. S. 1994, MNRAS, 268, 709

Buccheri, R., Bennett, K., Bignami, G. F., et al. 1983, A\&A, 128, 245

Buccheri, R., di Gesu, V., Maccarone, M. C., \& Sacco, B. 1988, A\&A, 201, 194

Corradi, R. L. M., Rodríguez-Flores, E. R., Mampaso, A., et al. 2008, A\&A, 480, 409

Cutri, R. M., Skrutskie, M. F., van Dyk, S., et al. 2012, VizieR Online Data Catalog: II/311

Cutri, R. M., Skrutskie, M. F., van Dyk, S., et al. 2003, VizieR Online Data Catalog: II/246

Ducci, L., Doroshenko, V., Suleimanov, V., et al. 2016, A\&A, 592, A58

Fabbiano, G. 2006, ARA\&A, 44, 323

Frebel, A., Kirby, E. N., \& Simon, J. D. 2010, Nature, 464, 72

Gaia Collaboration (Brown, A. G. A., et al.) 2018, A\&A, 616, A1

Geske, M. T., Gettel, S. J., \& McKay, T. A. 2006, AJ, 131, 633

Girardi, L., Grebel, E. K., Odenkirchen, M., \& Chiosi, C. 2004, A\&A, 422, 205

Güdel, M. 2004, A\&ARv, 12, 71

Kennea, J. A., Mukai, K., Sokoloski, J. L., et al. 2009, ApJ, 701, 1992

Kenyon, S. J., Livio, M., Mikolajewska, J., \& Tout, C. A. 1993, ApJ, 407, L81

Kirby, E. N., Simon, J. D., Geha, M., Guhathakurta, P., \& Frebel, A. 2008, ApJ, $685, \mathrm{~L} 43$
Kirby, E. N., Guhathakurta, P., Simon, J. D., et al. 2010, ApJS, 191, 352

Kirby, E. N., Guo, M., Zhang, A. J., et al. 2015, ApJ, 801, 125

Kleyna, J., Wilkinson, M. I., Evans, N. W., Gilmore, G., \& Frayn, C. 2002, MNRAS, 330, 792

Lewin, W. H. G., \& van der Klis, M. 2006, Compact Stellar X-ray Sources (Cambridge, UK: Cambridge University Press)

Liebert, J. 1994, in Cool Stars, Stellar Systems, and the Sun, ed. J. P. Caillault, ASP Conf. Ser., 64, 520

Lindegren, L., Hernández, J., Bombrun, A., et al. 2018, A\&A, 616, A2

Luna, G. J. M., \& Sokoloski, J. L. 2007, ApJ, 671, 741

Luna, G. J. M., Sokoloski, J. L., \& Mukai, K. 2008, in RS Ophiuchi (2006) and the Recurrent Nova Phenomenon, eds. A. Evans, M. F. Bode, T. J. O'Brien, \& M. J. Darnley, ASP Conf. Ser., 401, 342

Luna, G. J. M., Sokoloski, J. L., Mukai, K., \& Nelson, T. 2013, A\&A, 559, A6

Maccacaro, T., Gioia, I. M., Wolter, A., Zamorani, G., \& Stocke, J. T. 1988, ApJ, 326,680

Maccarone, T. J., Kundu, A., Zepf, S. E., Piro, A. L., \& Bildsten, L. 2005 , MNRAS, 364, L61

Mann, A. W., Feiden, G. A., Gaidos, E., Boyajian, T., \& von Braun, K. 2015, ApJ, 804, 64

Manni, L., Nucita, A. A., De Paolis, F., Testa, V., \& Ingrosso, G. 2015, MNRAS, 451, 2735

Mason, K. O., Breeveld, A., Much, R., et al. 2001, A\&A, 365, L36

Muerset, U., Wolff, B., \& Jordan, S. 1997, A\&A, 319, 201

Nuñez, N. E., Nelson, T., Mukai, K., Sokoloski, J. L., \& Luna, G. J. M. 2016, ApJ, 824, 23

Parker, E. N. 1975, ApJ, 198, 205

Piatek, S., Pryor, C., Armandroff, T. E., \& Olszewski, E. W. 2001, AJ, 121, 841

Pogson, N. 1856, MNRAS, 17, 12

Primini, F. A., Forman, W., \& Jones, C. 1993, ApJ, 410, 615

Ramsay, G., \& Wu, K. 2006, A\&A, 459, 777

Rave, H. A., Zhao, C., Newberg, H. J., et al. 2003, ApJS, 145, 245

Rosen, S. 2016, VizieR Online Data Catalog: Enhanced 3XMM catalogue (3XMMe), IX/047

Saeedi, S., Sasaki, M., \& Ducci, L. 2016, A\&A, 586, A64

Saeedi, S., Sasaki, M., \& Ducci, L. 2018, MNRAS, 473, 440

Scargle, J. D. 1982, ApJ, 263, 835

Schlafly, E. F., \& Finkbeiner, D. P. 2011, ApJ, 737, 103

Schlegel, D. J., Finkbeiner, D. P., \& Davis, M. 1998, ApJ, 500, 525

Ségall, M., Ibata, R. A., Irwin, M. J., Martin, N. F., \& Chapman, S. 2007, MNRAS, 375, 831

Shaw, J. S., Caillault, J.-P., \& Schmitt, J. H. M. M. 1996, ApJ, 461, 951

Sonbas, E., Rangelov, B., Kargaltsev, O., et al. 2016, ApJ, 821, 54

Stelzer, B., Marino, A., Micela, G., López-Santiago, J., \& Liefke, C. 2013, MNRAS, 431, 2063

Strüder, L., Briel, U., Dennerl, K., et al. 2001, A\&A, 365, L18

Suleimanov, V., Revnivtsev, M., \& Ritter, H. 2005, A\&A, 435, 191

Tauris, T. M., \& van den Heuvel, E. P. J. 2006, in Formation and Evolution of Compact Stellar X-ray Sources, eds. W. H. G. Lewin, \& M. van der Klis, 623

Tolstoy, E., Hill, V., \& Tosi, M. 2009, ARA\&A, 47, 371

Turner, M. J. L., Abbey, A., Arnaud, M., et al. 2001, A\&A, 365, L27

West, A. A., Walkowicz, L. M., \& Hawley, S. L. 2005, PASP, 117, 706

West, A. A., Morgan, D. P., Bochanski, J. J., et al. 2011, AJ, 141, 97

Wheatley, P. J., \& Kallman, T. R. 2006, MNRAS, 372, 1602

Yanny, B., Rockosi, C., Newberg, H. J., et al. 2009, AJ, 137, 4377 


\section{Appendix A: Long-term light curves}
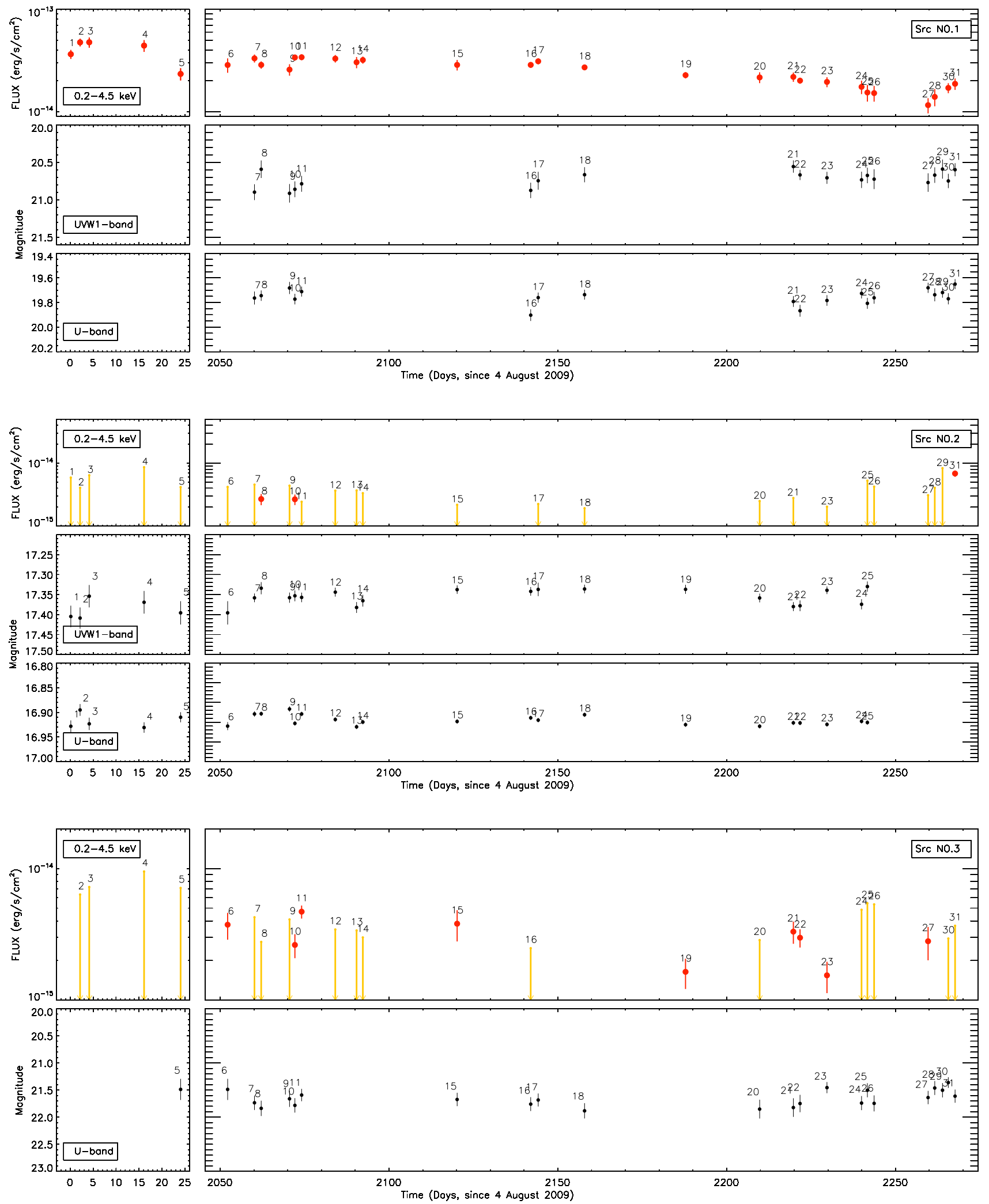

Fig. A.1. Light curve of each source over thirty-one XMM-Newton observations of EPIC cameras and OM cameras. The absorbed weighted flux $(0.2-4.5 \mathrm{keV})$ of each source in the different observations is plotted over time. If the source was not detected in an observation, the upper limit is shown. In the cases of X-ray light curves, the red dots show the flux of each observation and the orange arrows show the upper limits. In optical and UV light curves, the black dots show the magnitude of the source and the grey arrows show the upper limits. 
A\&A 627, A128 (2019)
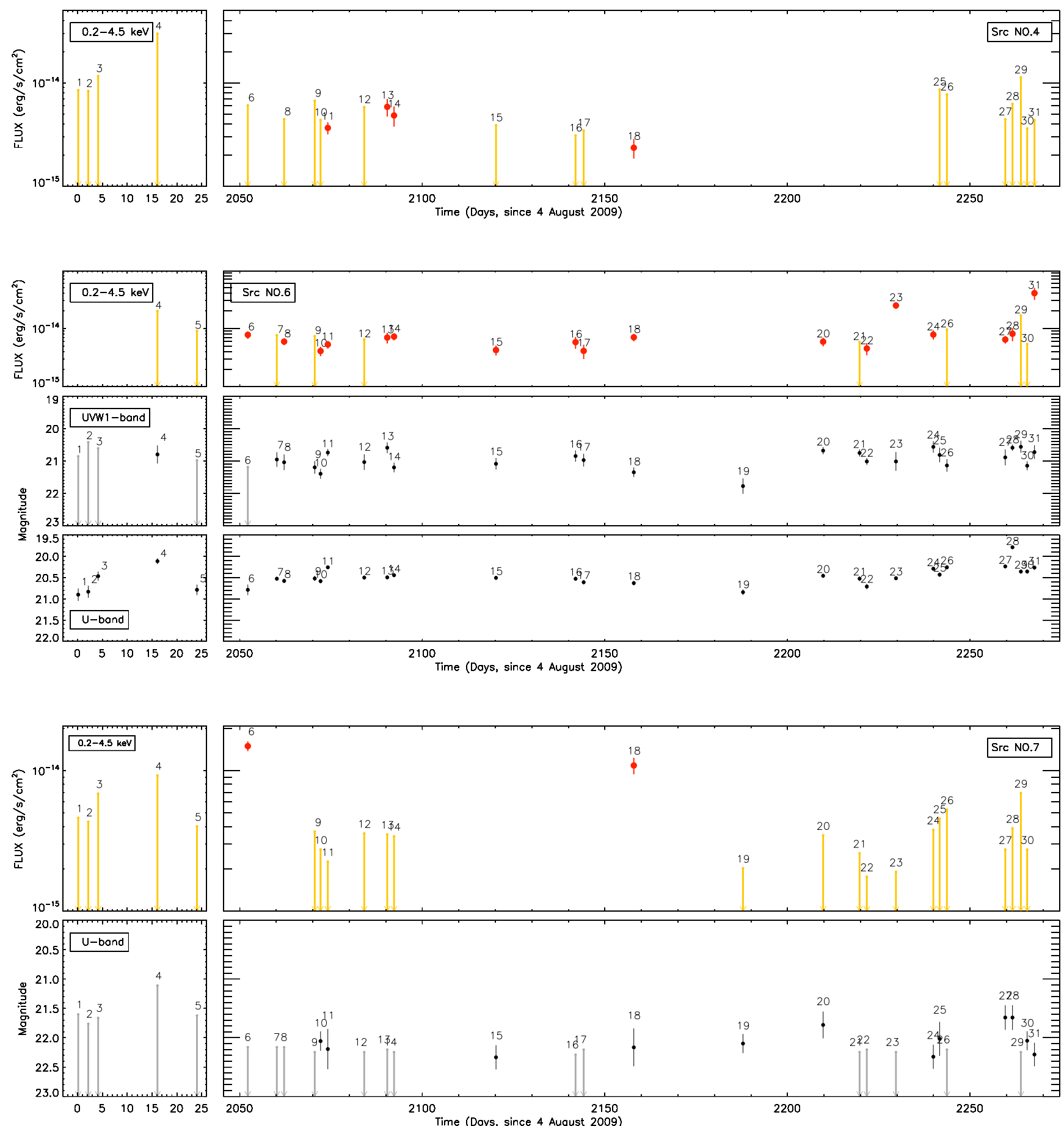

Fig. A.1. continued. 
S. Saeedi et al.: Classification of low luminosity stellar X-ray sources of the Draco dSph
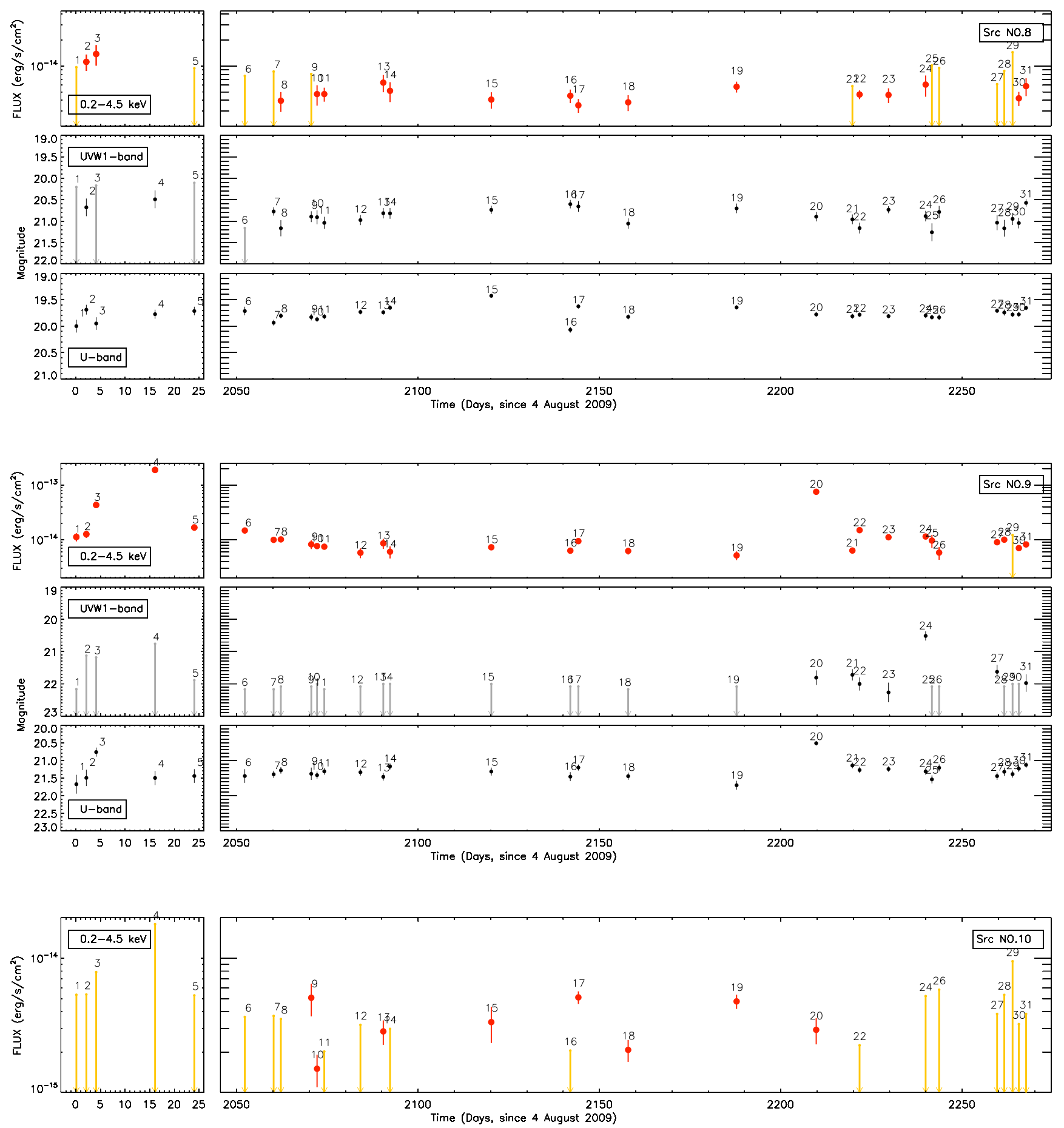

Fig. A.1. continued. 
A\&A 627, A128 (2019)
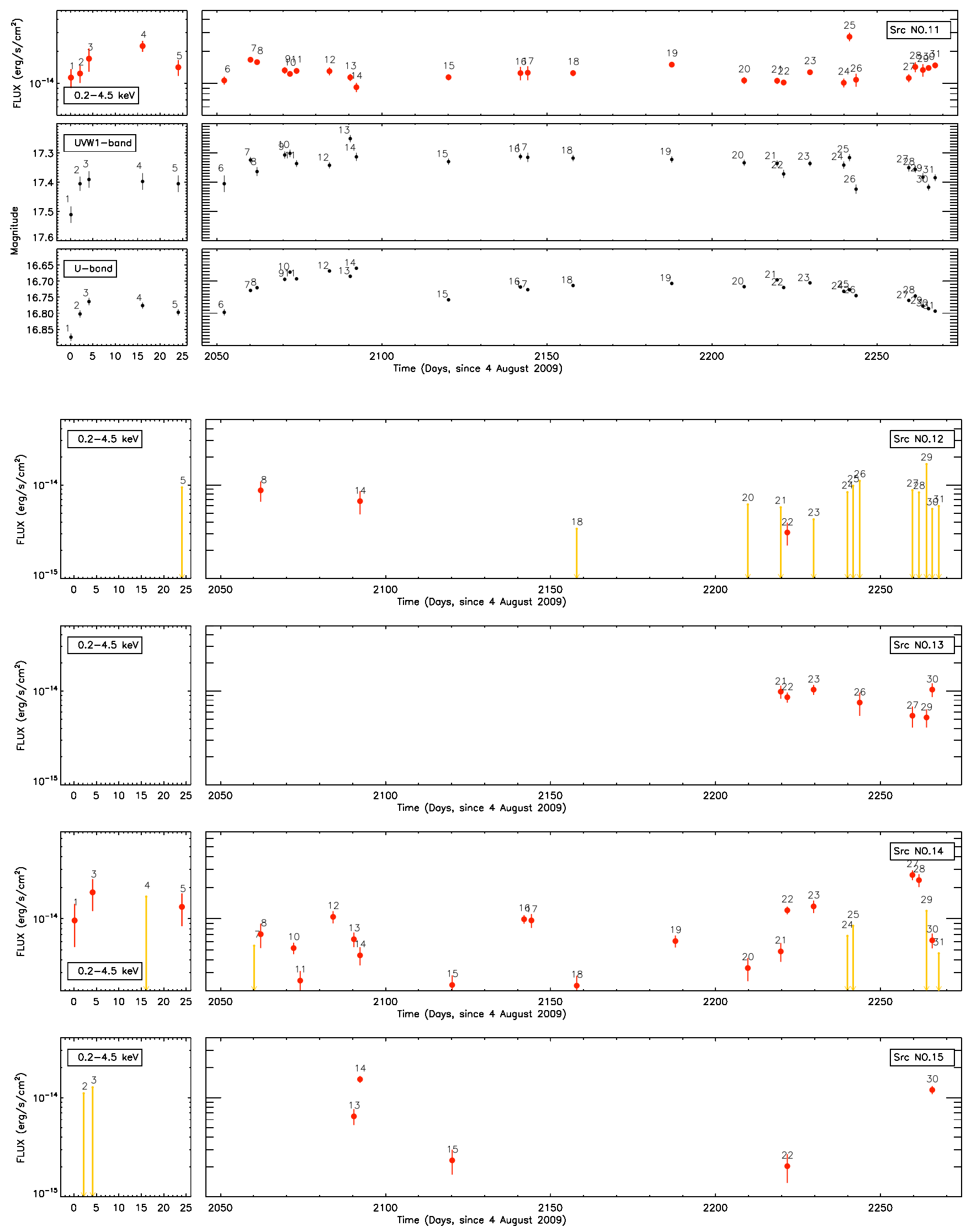

Fig. A.1. continued. 


\section{Appendix B: Optical and infrared magnitudes of the counterparts of X-ray sources}

Table B.1. Optical magnitudes of counterparts of X-ray sources in different energy filters of the SDSS9 survey.

\begin{tabular}{lccccc}
\hline \hline Src-No & $u \mathrm{mag}$ & $g \mathrm{mag}$ & $r \mathrm{mag}$ & $i \mathrm{mag}$ & $z \mathrm{mag}$ \\
\hline 1 & $20.64 \pm 0.05$ & $18.837 \pm 0.008$ & $18.028 \pm 0.006$ & $17.682 \pm 0.007$ & $17.49 \pm 0.02$ \\
2 & $17.75 \pm 0.01$ & $16.580 \pm 0.004$ & $16.133 \pm 0.004$ & $15.971 \pm 0.004$ & $15.91 \pm 0.01$ \\
3 & $22.58 \pm 0.27$ & $19.788 \pm 0.016$ & $18.225 \pm 0.007$ & $17.322 \pm 0.006$ & $16.82 \pm 0.01$ \\
4 & $18.05 \pm 0.01$ & $16.709 \pm 0.004$ & $16.223 \pm 0.004$ & $16.084 \pm 0.004$ & $16.05 \pm 0.01$ \\
5 & $19.08 \pm 0.02$ & $17.753 \pm 0.005$ & $16.476 \pm 0.004$ & $16.191 \pm 0.004$ & $15.77 \pm 0.01$ \\
6 & $21.34 \pm 0.10$ & $20.082 \pm 0.019$ & $19.426 \pm 0.014$ & $19.148 \pm 0.017$ & $19.06 \pm 0.05$ \\
7 & $23.79 \pm 0.65$ & $20.770 \pm 0.030$ & $19.246 \pm 0.013$ & $17.931 \pm 0.008$ & $17.24 \pm 0.01$ \\
8 & $20.69 \pm 0.06$ & $18.612 \pm 0.008$ & $17.746 \pm 0.006$ & $17.387 \pm 0.006$ & $17.17 \pm 0.01$ \\
9 & $22.06 \pm 0.17$ & $19.484 \pm 0.012$ & $17.985 \pm 0.006$ & $16.845 \pm 0.005$ & $16.21 \pm 0.01$ \\
10 & $23.79 \pm 0.66$ & $21.942 \pm 0.081$ & $20.417 \pm 0.030$ & $18.693 \pm 0.012$ & $17.73 \pm 0.02$ \\
11 & $17.59 \pm 0.01$ & $15.973 \pm 0.003$ & $15.301 \pm 0.003$ & $15.033 \pm 0.004$ & $14.88 \pm 0.00$ \\
12 & $22.95 \pm 0.39$ & $20.213 \pm 0.018$ & $18.690 \pm 0.009$ & $17.317 \pm 0.006$ & $16.55 \pm 0.01$ \\
13 & $24.80 \pm 1.07$ & $21.307 \pm 0.045$ & $19.887 \pm 0.022$ & $18.761 \pm 0.013$ & $18.13 \pm 0.02$ \\
14 & $22.00 \pm 0.20$ & $19.170 \pm 0.011$ & $17.647 \pm 0.006$ & $16.210 \pm 0.005$ & $15.42 \pm 0.01$ \\
15 & $21.93 \pm 0.17$ & $19.562 \pm 0.012$ & $18.115 \pm 0.007$ & $16.974 \pm 0.005$ & $16.36 \pm 0.01$ \\
\hline
\end{tabular}

Table B.2. Infrared magnitudes of counterparts of X-ray sources in different energy filters of 2MASS and WISE surveys.

\begin{tabular}{lccccccc}
\hline \hline Src-No & $J \mathrm{mag}$ & $H \mathrm{mag}$ & $K \mathrm{mag}$ & $W 1 \mathrm{mag}$ & $W 2 \mathrm{mag}$ & $W 3 \mathrm{mag}$ & $W 4 \mathrm{mag}$ \\
\hline 1 & $16.54 \pm 0.12$ & $15.74 \pm 0.14$ & $15.78 \pm 0.22$ & $15.49 \pm 0.04$ & $15.04 \pm 0.06$ & $12.46 \pm 0.25$ & $9.08 \pm 0.3$ \\
2 & $15.07 \pm 0.04$ & $14.64 \pm 0.07$ & $14.74 \pm 0.09$ & $14.57 \pm 0.03$ & $14.66 \pm 0.05$ & $<13.27^{(*)}$ & $<9.61^{(*)}$ \\
3 & $15.62 \pm 0.07$ & $14.93 \pm 0.08$ & $14.60 \pm 0.08$ & $14.52 \pm 0.15$ & $14.59 \pm 0.04$ & $<13.12^{(*)}$ & $<9.37^{(*)}$ \\
5 & $14.38 \pm 0.03$ & $13.71 \pm 0.04$ & $13.46 \pm 0.04$ & $13.25 \pm 0.11$ & $13.27 \pm 0.03$ & $12.49 \pm 0.29$ & $<9.51^{(*)}$ \\
6 & - & - & - & - & - & - & - \\
7 & $15.87 \pm 0.09$ & $15.10 \pm 0.09$ & $15.05 \pm 0.12$ & $14.60 \pm 0.03$ & $14.47 \pm 0.04$ & $<12.84^{(*)}$ & $<9.50^{(*)}$ \\
8 & $16.31 \pm 0.10$ & $15.68 \pm 0.13$ & $15.42 \pm 0.18$ & $15.35 \pm 0.04$ & $15.55 \pm 0.10$ & $<13.10^{(*)}$ & $<9.52^{(*)}$ \\
9 & $14.95 \pm 0.05$ & $14.40 \pm 0.06$ & $14.12 \pm 0.08$ & $13.93 \pm 0.10$ & $13.80 \pm 0.03$ & $12.81 \pm 0.42$ & $<9.32^{(*)}$ \\
10 & $16.16 \pm 0.12$ & $15.55 \pm 0.14$ & $15.09 \pm 0.18$ & $<15.06^{(*)}$ & $15.05 \pm 0.05$ & $<12.82^{(*)}$ & $<9.47^{(*)}$ \\
11 & $13.96 \pm 0.03$ & $13.43 \pm 0.03$ & $13.33 \pm 0.03$ & $13.27 \pm 0.03$ & $13.25 \pm 0.08$ & $13.05 \pm 0.42$ & $<9.51^{(*)}$ \\
12 & $15.05 \pm 0.04$ & $14.57 \pm 0.06$ & $14.30 \pm 0.06$ & $<14.19^{(*)}$ & $13.99 \pm 0.03$ & $<13.04^{(*)}$ & $<9.43^{(*)}$ \\
13 & $16.83 \pm 0.17$ & $16.09 \pm 0.19$ & $15.70 \pm 0.164$ & $15.46 \pm 0.04$ & $15.16 \pm 0.07$ & $<13.13^{(*)}$ & $<9.61^{(*)}$ \\
14 & $13.99 \pm 0.03$ & $13.40 \pm 0.03$ & $13.13 \pm 0.03$ & $12.97 \pm 0.03$ & $12.78 \pm 0.04$ & $12.51 \pm 0.27$ & $<9.62^{(*)}$ \\
15 & $15.01 \pm 0.05$ & $14.27 \pm 0.04$ & $14.11 \pm 0.06$ & $13.94 \pm 0.15$ & $13.84 \pm 0.03$ & $<12.89^{(*)}$ & $<9.50^{(*)}$ \\
\hline
\end{tabular}

Notes. ${ }^{(*)}$ Indicates that the upper limit is reported in the WISE catalogue. 


\section{Appendix C: Image of optical SDSS9 counterparts}

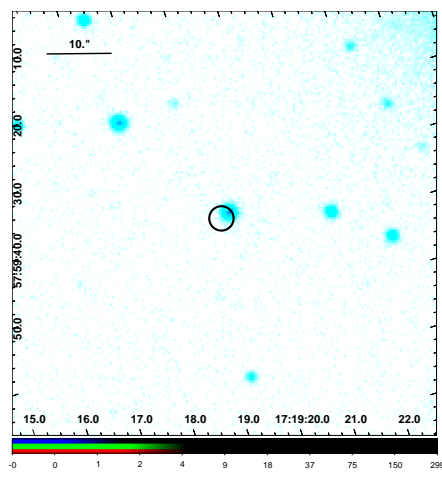

(a) Src No.1

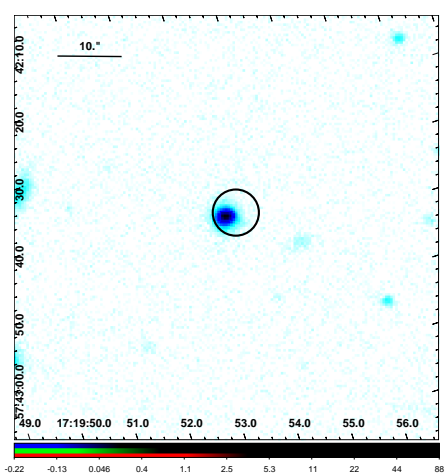

(d) Src No.4

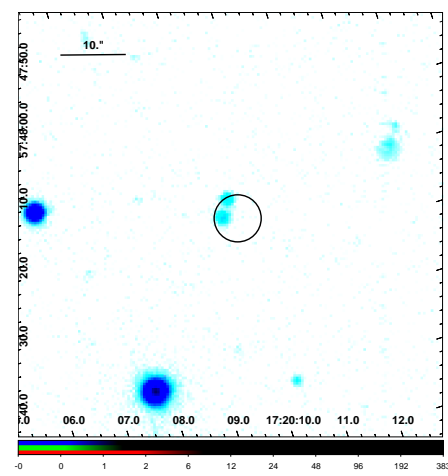

(g) Src No.7

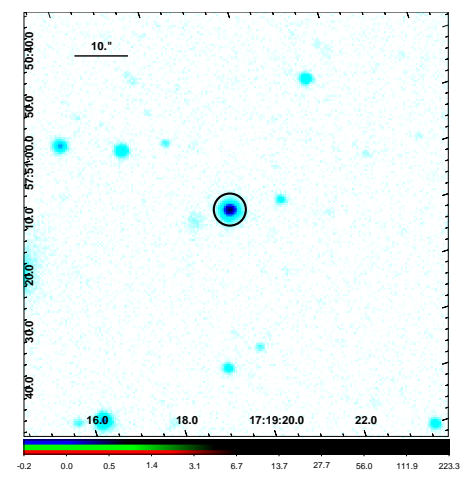

(b) Src No.2

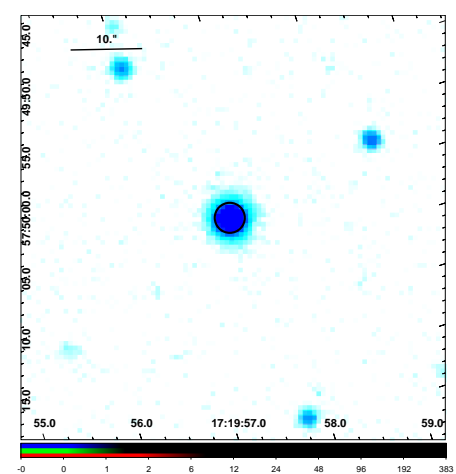

(e) Src No.5

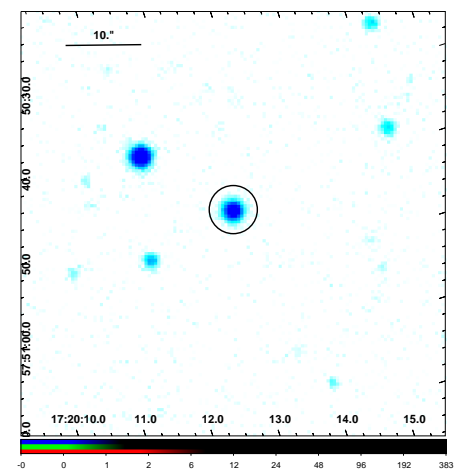

(h) Src No.8

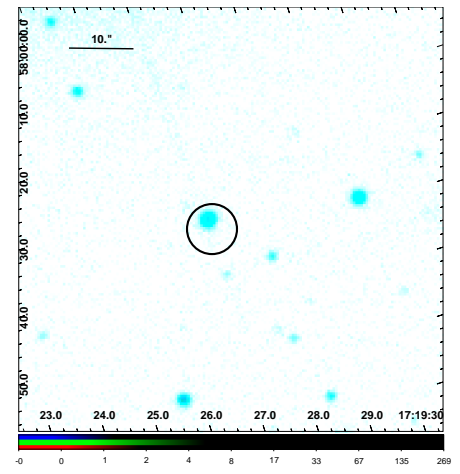

(c) Src No.3

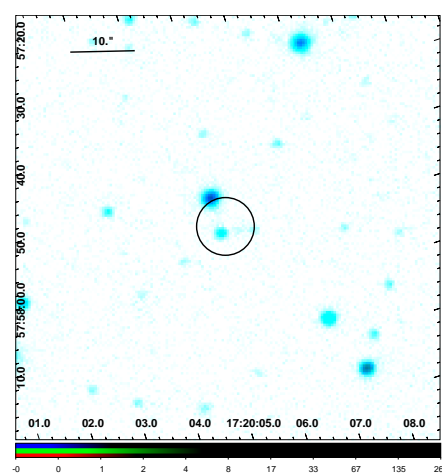

(f) Src No.6

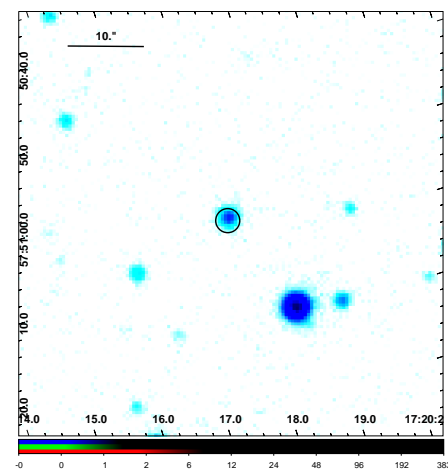

(i) Src No.9

Fig. C.1. Optical image of the counterpart of the X-ray sources from the SDSS9 survey. The black circles show the $3 \sigma$ error of X-ray source positions. 
S. Saeedi et al.: Classification of low luminosity stellar X-ray sources of the Draco dSph

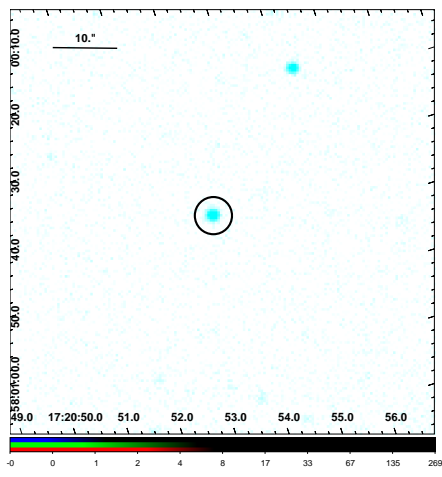

(a) Src No.10

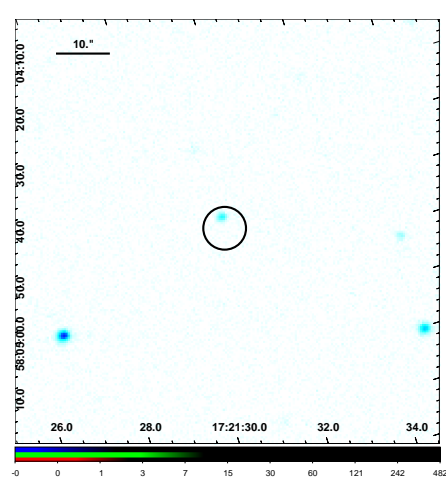

(d) Src No.13

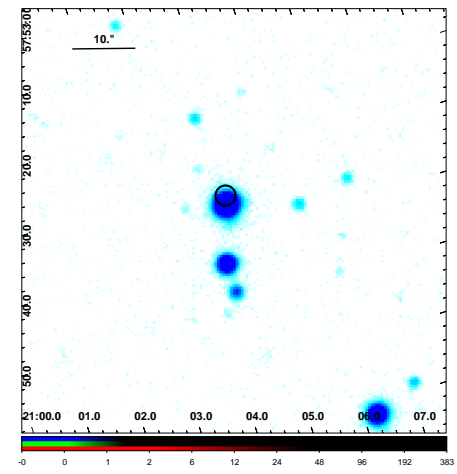

(b) Src No.11

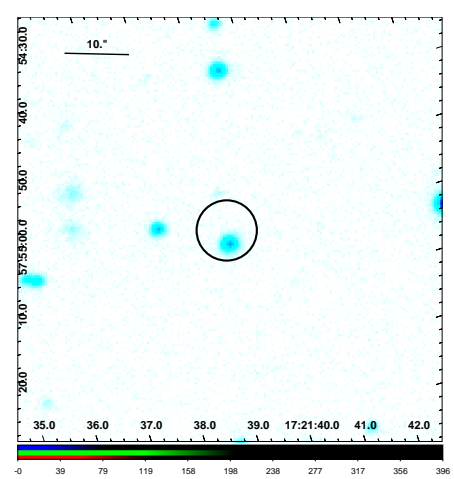

(e) Src No.14

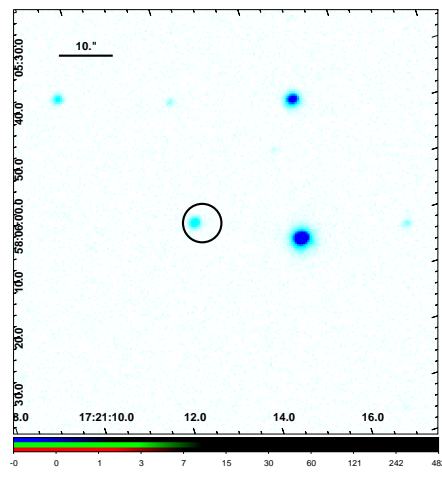

(c) Src No.12

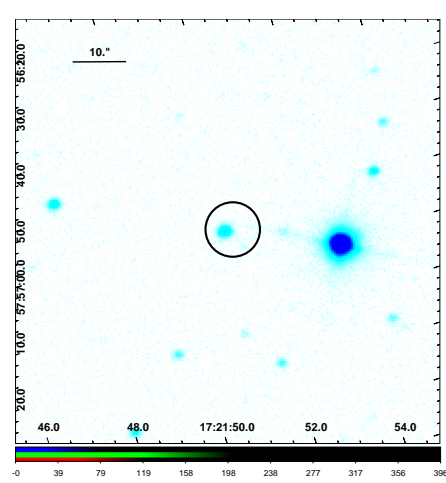

(f) $\operatorname{Src}$ No.15

Fig. C.1. continued. 